\title{
Estudios MORFO-ANATÓMICOS DE DOMACIOS FOLIARES EN RUBIÁCEAS ARGENTINAS
}

\author{
MARÍA FLORENCIA ROMERO1', ROBERTO SALAS y ANA MARIA GONZALEZ
}

\begin{abstract}
Summary: Morpho-anatomic studies of leaf domatia in Argentinian Rubiaceae. This paper deals with the study of leaf domatia in Argentine Rubiaceae, which are common morphogenetic structures on the underside of the leaves of woody plants, which can establish mutual relations with mites or ants. Tufts of hair, pockets, cavities and crypts: many families are classified into types common relatively well preserved. Around 290 families of "dicots" have domatia, Rubiaceae being the best represented with all known and transitional types. Domestic classification by molecular data, Rubiaceae divided into three subfamilies: Rubioideae, Ixorideae and Cinchonoideae. In Argentina inhabit 41 genera and 132 species of Rubiaceae, of which 34 are woody (27\%). As result, tree domatia types were found: tuft of hair, pockets and crypt, present in 14 species of Rubiaceace. It is mentioned for the first time a domatia in a vine species of Manettia. The results were compared with recent molecular studies; this confirmed the plesiomorphic condition thereof, since different types are common to the three subfamilies almost all the tribes of the family. Based on this sampling, the following tribes completely lack domatia: Anthospermeae, Rubieae, Paederieae and Pavetteae.
\end{abstract}

Key words: Anatomy, domatia, idioblasts, mites, Rubiaceae.

Resumen: Este trabajo estudia los domacios foliares en Rubiáceas argentinas, los cuales son estructuras morfogenéticas frecuentes en el envés de las hojas de plantas leñosas, que permiten establecer relaciones mutualistas con ácaros u hormigas. Se clasifican en tipos relativamente bien conservados comunes a numerosas familias: mechones de pelos, bolsillos, cavidades y criptas. Alrededor de 290 familias de "dicotiledóneas" tienen domacios, siendo Rubiaceae la mejor representada con todos los tipos conocidos y transicionales. La clasificación intrafamiliar según datos moleculares, divide a Rubiaceae en tres subfamilias: Rubioideae, Ixorideae y Cinchonoideae. En Argentina viven 41 géneros y 132 especies de Rubiáceas, de las cuales 34 son leñosas (27\%). Como resultado se encontraron tres tipos de domacios: mechón de pelos, bolsillos y en cripta en 14 especies de Rubiáceas. Se menciona por primera vez domacios en una enredadera sufrútice de la familia, en el género Manettia. Los resultados obtenidos fueron contrastados con estudios moleculares recientes, lo que permitió confirmar la condición plesiomórfica de los mismos, ya que los diferentes tipos son comunes a las tres subfamilias y a casi todas las tribus de la familia. Basado en el presente muestreo, las tribus Anthospermeae, Rubieae, Paederieae y Pavetteae carecen completamente de domacios.

Palabras clave: Ácaros, anatomía, domacios, idioblastos, Rubiaceae.

\section{INTRODUCCIÓN}

Los domacios foliares son estructuras que se encuentran frecuentemente en la cara abaxial de las hojas de numerosas plantas leñosas, particularmente las que habitan bosques tropicales o subtropicales

Instituto de Botánica del Nordeste (CONICET-UNNE), Sgto. Cabral 2131, 3400, Corrientes, Argentina.

${ }^{1}$ E-mail: mariafloromero@gmail.com
(Wilkinson, 1979). Funcionalmente los domacios representan una relación mutualista entre una planta y ácaros (acarodomacios) u hormigas (mirmecodomacios). Los domacios son estructuras morfogenéticas, por lo que su formación en la planta es independiente a la presencia del animal hospedante (Barros, 1961; Wilkinson, 1979; Brouwer \& Clifford, 1990). En base a este concepto, ácaros u hormigas serían ocupantes circunstanciales de estos refugios.

Algunos autores restringen el término domacio solo a aquellas formaciones presentes en la lámina 
foliar (Jacobs, 1966; Robbrecht, 1988), mientras que otros extienden el término a cavidades que se encuentran en tallos o pecíolos (Maschwitz et al., 1989; Tepe et al., 2007). En las hojas se encuentran especialmente en las axilas entre la vena principal y las secundarias, aunque también en nervaduras terciarias (Barros, 1959, 1961, 1962).

Estas estructuras suelen adoptar formas muy variadas, aunque algunos tipos son relativamente bien conservados y comunes a numerosas Eudicotiledóneas. Wilkinson (1979) clasificó a los domacios en cinco tipos: 1) mechones de pelos, 2) bolsas o bolsillos, 3) sacos, 4) criptas o cavidades y 5) márgenes revolutos, mientras que Robbrecht (1988) especificó para los domacios de Rubiáceas los tipos: mechones de pelos, bolsillos, cavidades, criptas y formas intermedias o combinaciones. De acuerdo a Brouwer \& Clifford (1990), unas 290 familias de Dicotiledóneas leñosas tienen domacios, entre las cuales la familia Rubiaceae es la que cuenta con el mayor número de especies con estas estructuras (Jacobs, 1966).

Los estudios recientes de domacios en la familia Rubiaceae se han centrado en especies con valor económico como el café, enfocándose en la evaluación del potencial benéfico o dañino de los ácaros hospedantes (O’Dowd, 1994; Nakamura et al., 2002; Mattos et al., 2004, 2006; Vega et al., 2007; Mineiro et al., 2008; Romero et al., 2011). Otros estudios de domacios en géneros americanos de Rubiaceae fueron realizados en Psychotria (Moraes et al., 2011a), Rudgea (Moraes et al., 2011b) y Simira (Moraes et al., 2009). La contribución más importante pertenece a Barros $(1959,1962)$, quien analizó 622 especies de Rubiáceas herbáceas y leñosas, encontrando domacios en 86 ejemplares leñosos.

En Argentina viven 41 géneros y 132 especies de Rubiáceas (Bacigalupo et al., 2008), principalmente distribuidas en las selvas Paranaense y de Las Yungas. En esta familia se utiliza el tipo de domacio como un carácter taxonómico para diferenciar especies, esto apoyado principalmente en su carácter morfogenético (Robbrecht, 1979, 1987; Nakamura et al., 2002). Sin embargo, en diversas familias se propuso evaluar cuidadosamente el valor taxonómico de los domacios, debido a que en algunas especies su presencia y tipo son variables (Jacobs, 1966; Robbrecht, 1988; Gonzalez, 2011).

Los objetivos de este trabajo son (1) determinar la presencia de domacios en las especies argentinas de Rubiaceae, (2) analizar la anatomía de los domacios presentes en las especies estudiadas, (3) tipificar las especies según las diferentes clases de domacios y (4) revaluar su aplicación en la taxonomía en algunos géneros de la familia.

\section{Materiales y Métodos}

\section{Estudios morfológicos}

Se analizó material fresco (cultivado), fijado en FAA (alcohol $70^{\circ}$, ác. acético y formol, 90:5:5) y material proveniente de ejemplares del herbario, principalmente del Herbario Carmen L. Cristóbal del Instituto de Botánica del Nordeste (CTES), estos últimos fueron preferentemente los mejores conservados y recientemente colectados. Se estudiaron un total de 132 especies, de hábitos leñosos y herbáceos, de las que se analizaron un mínimo de 10 ejemplares por especie a fin de determinar presencia/ ausencia de domacios. En el caso de Manettia cordifolia Mart. se analizaron todos los ejemplares disponibles en el herbario CTES (150 especímenes) dada la baja frecuencia de aparición de esta estructura en observaciones a campo. Los estudios se realizaron mediante microscopio estereoscópico provisto de cámara digital y tubo de dibujo para el registro de las observaciones.

La lista completa de material estudiado figura en el Apéndice.

\section{Estudios anatómicos}

Una vez que se registró presencia/ausencia y tipos de domacios, se seleccionaron 9 especies para el estudio anatómico. Para ello se eligieron hojas sanas de tamaño adulto, las cuales fueron procesadas para ser estudiadas mediante microscopía óptica (MO) y electrónica de barrido (MEB). Se realizaron cortes seriados para lo cual el material se incluyó en parafina según técnica de Johansen (1940) modificada por Gonzalez \& Cristóbal (1997). Se realizaron cortes transversales (CT), con micrótomo rotativo, de $12 \mu \mathrm{m}$ de espesor. Se empleó una doble tinción safranina-Astra blue (Luque et al., 1996). Los cortes fueron montados en bálsamo de Canadá sintético. Las observaciones y fotografías se realizaron con microscopio Leica DM LB2 equipado con cámara fotográfica.

Además se efectuaron observaciones en vista superficial con Microscopio Electrónico de Barrido, para lo cual se empleó material fijado en FAA, 
posteriormente fue secado a punto crítico y metalizado con Oro-Paladio. Se utilizó el MEB Jeol LV 5800 del Servicio de Microscopía Electrónica de la UNNE, Corrientes.

A continuación se enumeran las nueve especies que fueron coleccionadas para el estudio morfoanatómico, las que se encuentran depositadas en el herbario CTES:

Calycophyllum multiflorum Griseb.: Prov. Formosa: Dpto. Ingeniero Juárez, $3 \mathrm{~km}$ pasando el pueblo, 18-III-2014, Salas 497.

Chomelia obtusa Cham. \& Schltdl.: Prov. Misiones: Dpto. San Pedro, Parque Provincial Moconá, 07III-2013, Judkevich 47.

Coussarea contracta (Walp.) Müll. Arg.: Prov. Misiones: Parque Provincial Cruce Caballero, Sendero Curi-y, 26³1'08'S, 5359'20,2”W, 05III-2013, Judkevich 30.

Coutarea hexandra (Jacq.) K. Schum.: Prov. Salta:
Dpto. Aguaray, 20²0'47,4”S, 6348'11'W, 10XII-2012, Sato 201.

Guettarda uruguensis Cham. \& Schltdl.: Prov. Corrientes: Dpto. Santo Tomé, Ruta 40, camino a Colonia Pellegrini, 28 $25^{\prime} 02,16^{\prime} \mathrm{S}, 56^{\circ} 32^{\prime} 33^{\prime \prime} \mathrm{W}$, 24-I-2014, Florentín 1.

Machaonia brasiliensis (Humb.) Cham. \& Schltdl.:

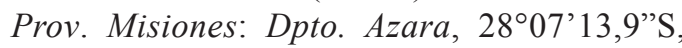
557'19,9'”, 28-II-2013, Judkevich 9.

Manettia cordifolia Mart.: Prov. Misiones: Dpto.

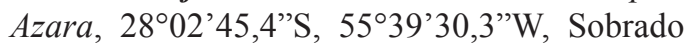
133.

Psychotria carthagenensis Jacq.: Prov. Corrientes: Dpto. Santo Tomé, 2825’02,16”S, 56³2’33” W, 24-I-2014, Florentín 6.

Randia ferox (Cham. \& Schltdl.) DC.: Prov. Misiones: Dpto. San Pedro, Parque Provincial Moconá, mirador del Yaboty, 2709'03,67’S, 5359'51,09’W, 07-III-2013, Judkevich 34.

Tabla 1. Listas de especies de Rubiáceas con domacios foliares

\begin{tabular}{|c|c|c|}
\hline Especie & $\begin{array}{l}\text { Tipo de domacio hallado } \\
\text { en el presente estudio }\end{array}$ & Tipo de domacio según bibliografía consultada \\
\hline $\begin{array}{l}\text { 1. Calycophyllum } \\
\text { multiflorum }\end{array}$ & Mechón de pelos & $\begin{array}{l}\text { "Pelos axilares" (Bacigalupo, 1993); Barros (1962) cita } \\
\text { "cripta" para C. spruceanum (Benth.) Hook. f. ex K. Schum. }\end{array}$ \\
\hline $\begin{array}{l}\text { 2. Cephalanthus } \\
\text { glabratus }\end{array}$ & $\begin{array}{l}\text { Domacios ausentes. } \\
\text { Ejemplar completamente } \\
\text { glabro }\end{array}$ & $\begin{array}{l}\text { Presencia dudosa, citado como "axilas barbadas" } \\
\text { (Bacigalupo, 1974); “Aglomerado de pelos" (Barros, 1959) }\end{array}$ \\
\hline 3. Chomelia obtusa & Cripta con pelos & $\begin{array}{l}\text { "Axilas barbadas" (Delprete et al., 2004); } \\
\text { "aglomerado de pelos" (Barros, 1959); "mechón } \\
\text { de pelos" (Barros, 1962; Delprete, 2010) }\end{array}$ \\
\hline 4. Cordiera concolor & Cripta con pelos & "Cripta" (Barros, 1959) \\
\hline 5. Coussarea contracta & Cripta con pelos internos & $\begin{array}{l}\text { Domacios presentes sin aclarar tipo (Gomes, 2007; Delprete et } \\
\text { al., 2004; Delprete, 2010). "Hoyo" (Brouwer \& Clifford, 1990) }\end{array}$ \\
\hline 6. Coutarea hexandra & Bolsillo con pelos & $\begin{array}{l}\text { "Aglomerado de pelos" (Barros, 1959); "axilas barbadas" } \\
\text { (Delprete, 2010); "axilas pubescentes" (Bacigalupo, 1993) }\end{array}$ \\
\hline 7. Guettarda uruguensis & Mechón de pelos & Primer registro para la especie \\
\hline $\begin{array}{l}\text { 7. Machaonia } \\
\text { brasiliensis }\end{array}$ & Bolsillo con pelos & Primer registro para la especie \\
\hline 8. Manettia cordifolia & Cripta con pelos & Primer registro para la especie \\
\hline 9. Palicourea crocea & Mechón de pelos & Primer registro para la especie \\
\hline $\begin{array}{l}\text { 10. Pogonopus } \\
\text { tubulosus }\end{array}$ & Mechón de pelos & "Mechón de pelos laxos" (Delprete, 1999) \\
\hline $\begin{array}{l}\text { 11. Psychotria } \\
\text { carthagenensis }\end{array}$ & $\begin{array}{l}\text { Bolsillo con mechón } \\
\text { de pelos }\end{array}$ & $\begin{array}{l}\text { Domacios presentes sin aclarar tipo } \\
\text { (Delprete, 2010; Moraes et al., 2011a) }\end{array}$ \\
\hline 12. Psychotria deflexa & Domacios ausentes & Domacios presentes sin aclarar tipo (Moraes et al., 2011a) \\
\hline 13. Randia ferox & Mechón de pelos & $\begin{array}{l}\text { "Mechón de pelos" } \\
\text { (Judkevich, 2013, Inéd.) }\end{array}$ \\
\hline 14. Rudgea parquioides & Cripta con pelos & "Hoyo" (Brouwer \& Clifford, 1990) \\
\hline 15. Simira sampoiana & Mechón de pelos & $\begin{array}{l}\text { "Mechón de pelos" (Bacigalupo \& Cabral, } \\
\text { 2003; Moraes et al., 2009) }\end{array}$ \\
\hline
\end{tabular}


Bol. Soc. Argent. Bot. 50 (4) 2015
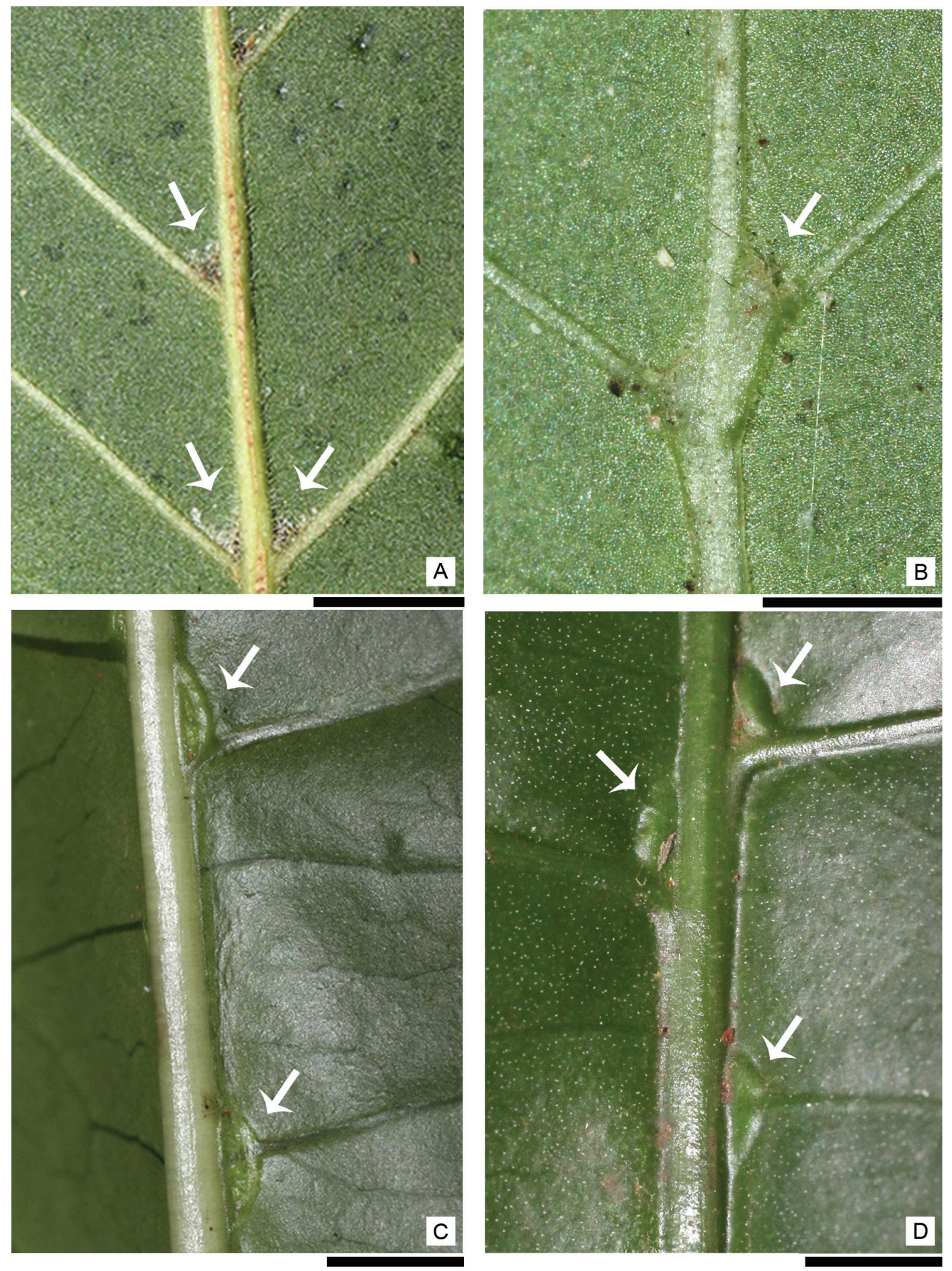

Fig. 1. Fotografías de tipos de domacios en vista superficial. A: domacio en mechón de pelos (flechas) en Randia ferox. B: domacio en bolsillo (flechas) en Psychotria carthagenensis. C-D: domacio en cripta (flechas) en Coussarea contracta. A-C: domacios en el envés de la lámina. D: domacio en el haz de la lámina. Escalas: A-D: 0,5 cm. 


\section{Resultados}

De las 132 especies de Rubiáceas estudiadas se encontraron 14 especies que presentan domacios, entre las que se observaron los siguientes tipos: mechones de pelos, bolsillos y criptas (Fig. 1).

En tabla 1 se registran todas las especies analizadas con domacios, comparándose con el tipo de domacio descripto en la bibliografía.

\section{Estudios morfo-anatómicos}

Del total de especies analizadas seleccionamos nueve taxones representativos con diversos tipos de domacios para realizar el estudio anatómico. Las características anatómicas de la lámina foliar y del domacio de las especies estudiadas se encuentran en la tabla 2. Los domacios presentes son de tres tipos:

Mechón de pelos: este domacio está formado por concentraciones de tricomas en el envés foliar, en la axila entre la vena principal y las secundarias. Los tricomas son simples, eglandulares y se entrelazan entre las costillas formadas por las venas principal y secundarias (Fig. 2 A-C). En el haz de la hoja, en correspondencia al domacio, puede presentarse ocasionalmente un pequeño domo. Este tipo de domacio en mechón de pelos fue hallado en Calycophyllum multiflorum y Randia ferox. En Guettarda uruguensis la presencia del domacio es variable; hay hojas con abundantes tricomas en toda la longitud de las venas, esta característica hace que el mechón de pelos entre venas que forma el domacio sea poco evidente.

Anatomía: el mesofilo en la zona del domacio mantiene las mismas características que en el resto de la lámina foliar. El mesofilo es dorsiventral con presencia de idioblastos con cristales, dependiendo de la especie son drusas o arena cristalina (Fig. 2 D-F, Tabla 2).

Bolsillo: este domacio estructuralmente posee un "piso" formado por la lámina foliar y un "techo" que es una expansión de tejido parenquimático que conecta la vena principal y una secundaria. En el envés se aprecia una bolsa de abertura amplia (Fig. 3 B-C) o simplemente la abertura distal (Fig. 3 A, D-E, G-I). Puede tener o no tricomas en el borde del bolsillo, entrelazándose para cubrir la abertura (Fig. 3 A-E, G-I). En el haz se presenta un domo correspondiente a la zona del domacio (Fig. 3 F). El domacio en bolsillo se encontró en Coutarea hexandra, Machaonia brasiliensis y Psychotria carthagenensis.

Anatomía: el piso presenta las mismas características que el resto de la lámina foliar. En las especies estudiadas el mesofilo es dorsiventral. La epidermis superior puede ser uni-bistratificada con presencia de tricomas según sea la especie (Fig. 5 A-D, Tabla 2). La epidermis inferior carece de estomas, y presenta tricomas. En las especies estudiadas el techo del domacio es homogéneo, está formado por parénquima clorofiliano compacto, sin diferenciación de parénquima en empalizada y lagunoso, las células son de forma redondeada, sin desarrollo de espacios intercelulares.

Cripta: es una cavidad constituida por un "piso" o base del domacio que se encuentra formada por la lámina foliar y el "techo" que es la expansión de tejido que conecta la vena principal y una secundaria. A diferencia del domacio en bolsillo, la abertura es circular o elíptica estrecha. La cripta puede ser glabra o presentar tricomas rodeando la abertura y/o en su interior (Fig. 4 A, D, E, G, H). Se caracteriza por un domo en el haz en la región del domacio (Fig. 4 B-C). El domacio en cripta fue hallado en Coussarea contracta, Chomelia obtusa y Manettia cordifolia.

Anatomía: el piso presenta las mismas características que el resto de la lámina foliar (Fig. 5 E-G, J, K). El mesofilo es dorsiventral variando el número de estratos del parénquima en empalizada entre las especies (Tabla 2). El mesofilo presenta drusas, rafidios o cristales romboidales según la especie (Fig. $5 \mathrm{H}, \mathrm{I})$. Presenta una epidermis superior uni o bistratificada, dependiendo de la especie y una epidermis inferior unistratificada con presencia de tricomas en las tres especies (Tabla 2). El techo del domacio es homogéneo en las especies estudiadas y está formado por parénquima clorofiliano compacto, sin diferenciación de parénquima en empalizada y lagunoso (Fig. 5 A-B, E, J, Tabla 2).

Indumento: los tricomas que forman los diversos tipos de domacio son simples y eglandulares en todas las especies (Tabla 2), siendo los mismos que tapizan la lámina, variando su densidad 
al constituir el domacio o cubrir su abertura. Estructuralmente se distinguen los siguientes tricomas:

- Unicelulares: están formados por una sola célula larga que se inserta a la epidermis al mismo nivel que las restantes células epidérmicas. De acuerdo a la longitud se identifican tricomas cortos o largos. Los tricomas cortos tienen una longitud promedio de $62 \mu \mathrm{m}$ (46-75 $\mu \mathrm{m}$ ) (Figs. 3 B-C; 4 A, G). Los tricomas largos presentan una longitud promedio de $518 \mu \mathrm{m}$ (367-657 $\mu \mathrm{m})$ (Figs. $2 \mathrm{D} ; 4 \mathrm{~A}$ ).

- Uniseriados: tricomas formados por un pie que se inserta al mismo nivel que las células epidérmicas y un cuerpo formado por 3 a 6 células dispuestas en una serie. También se distinguen por su longitud, los tricomas cortos presentan una longitud promedio de $116 \mu \mathrm{m}$ (100-144 $\mu \mathrm{m})$ (Fig. 3 B-C). Los tricomas largos se corresponden con una longitud promedio de $447 \mu \mathrm{m}(299-575 \mu \mathrm{m})$ (Figs. $2 \mathrm{~A}$; 3 G-I; 4 D-E).

Los distintos tipos de tricomas presentan paredes lignificadas y el extremo apical es aguzado. Los tricomas cortos adoptan formas rectas y los tricomas largos formas curvas. Todos están cubiertos por una cutícula lisa (Figs. 3-4).

La presencia de ácaros y/o sus huevos entre los tricomas (Figs. 3 D-E; 4 G, I) expondría la función del domacio: brindar un hospedaje a estos ocupantes circunstanciales en condiciones ambientales adversas, evidenciando una relación mutualista entre los ácaros y la planta.
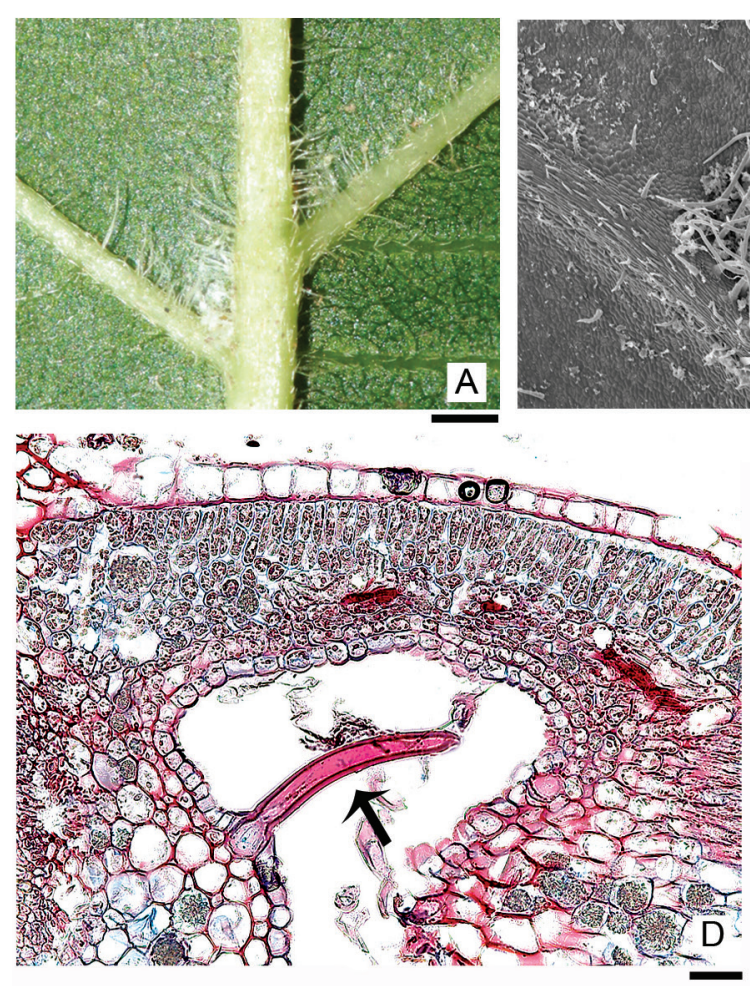
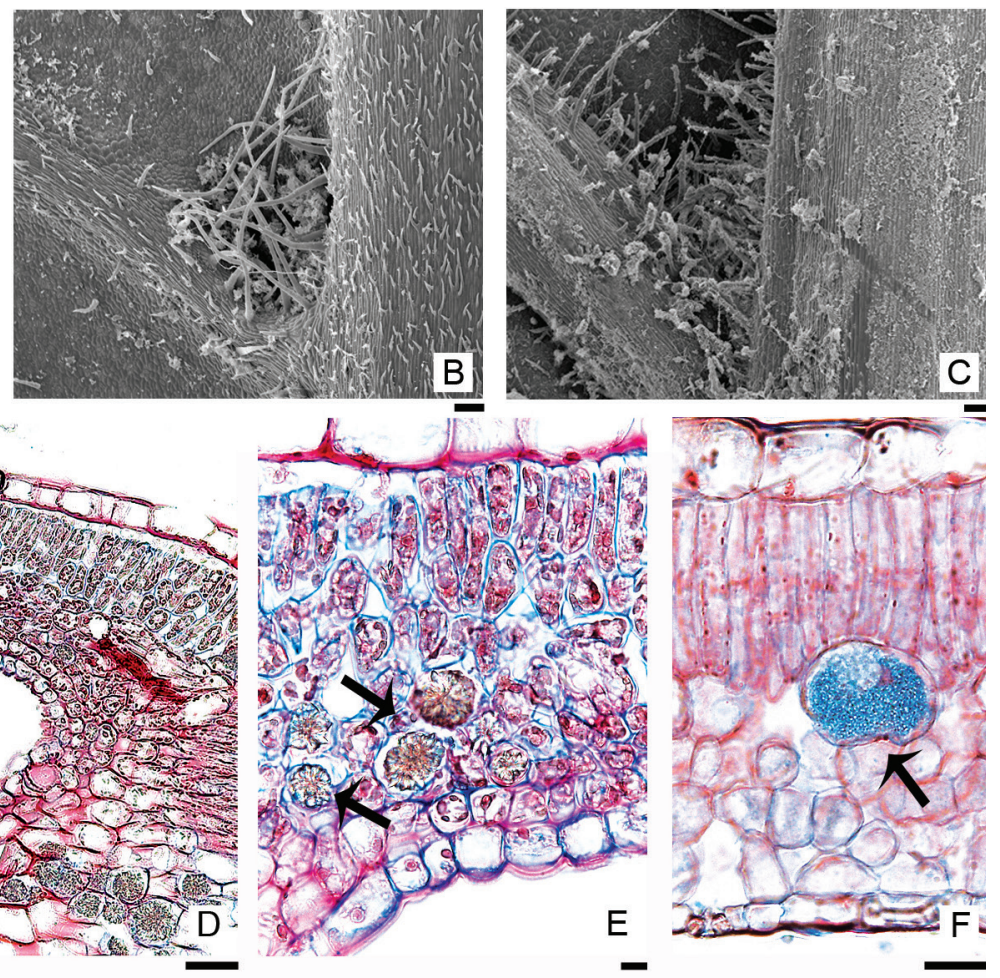

Fig. 2. Especies con domacios en mechón de pelos. A: fotografía de domacio en vista superficial; B-C: vista superficial con MEB, D-F: CT con MO. A: Guettarda uruguensis, envés mostrando conexión entre la vena principal y secundaria con tricomas tipo uniseriados largos formando el domacio. B, F: Calycophyllum multiflorum, B: domacio en envés foliar. F: lámina con idioblastos con arena cristalina (flecha). C-E: Randia ferox, C: domacio en envés foliar. D: domacio con tricoma unicelular largo (flecha). E: detalle de los idioblastos con drusas en el mesofilo (flecha). Escalas: A: 0,5 mm; B-C: $100 \mu \mathrm{m}$; D: $50 \mu \mathrm{m}$; E: $10 \mu \mathrm{m}$; F: $20 \mu \mathrm{m}$. 


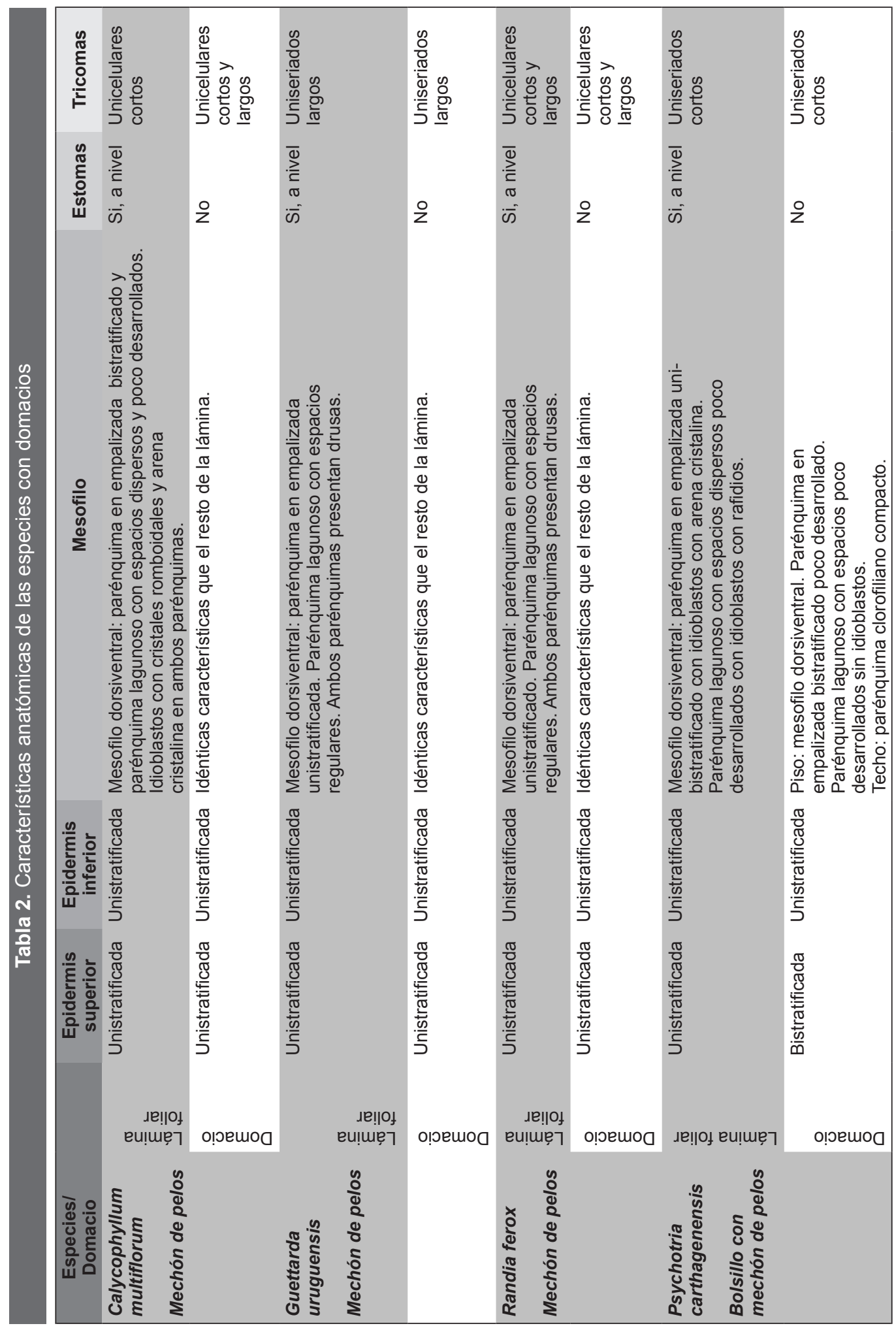


Bol. Soc. Argent. Bot. 50 (4) 2015

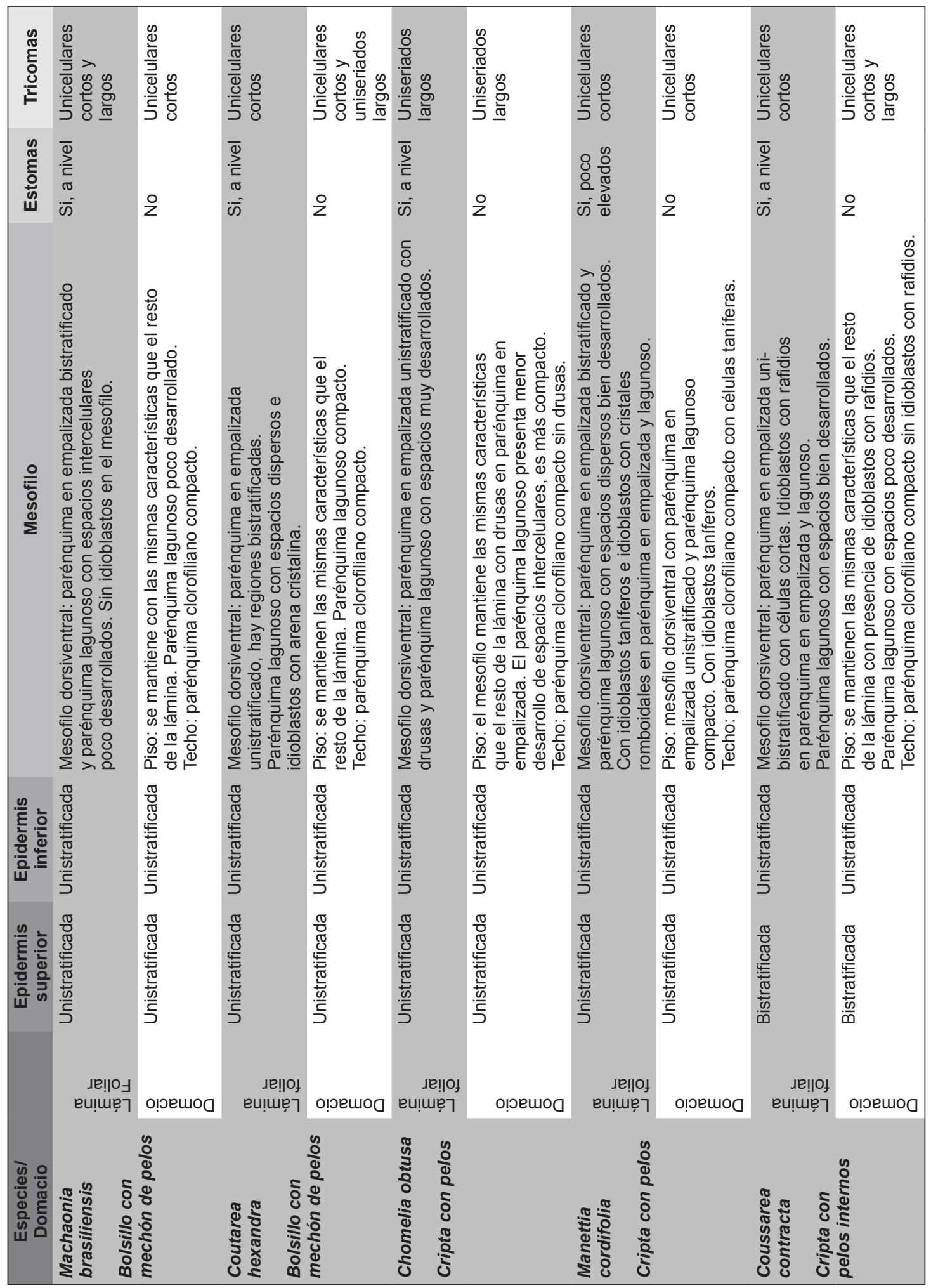



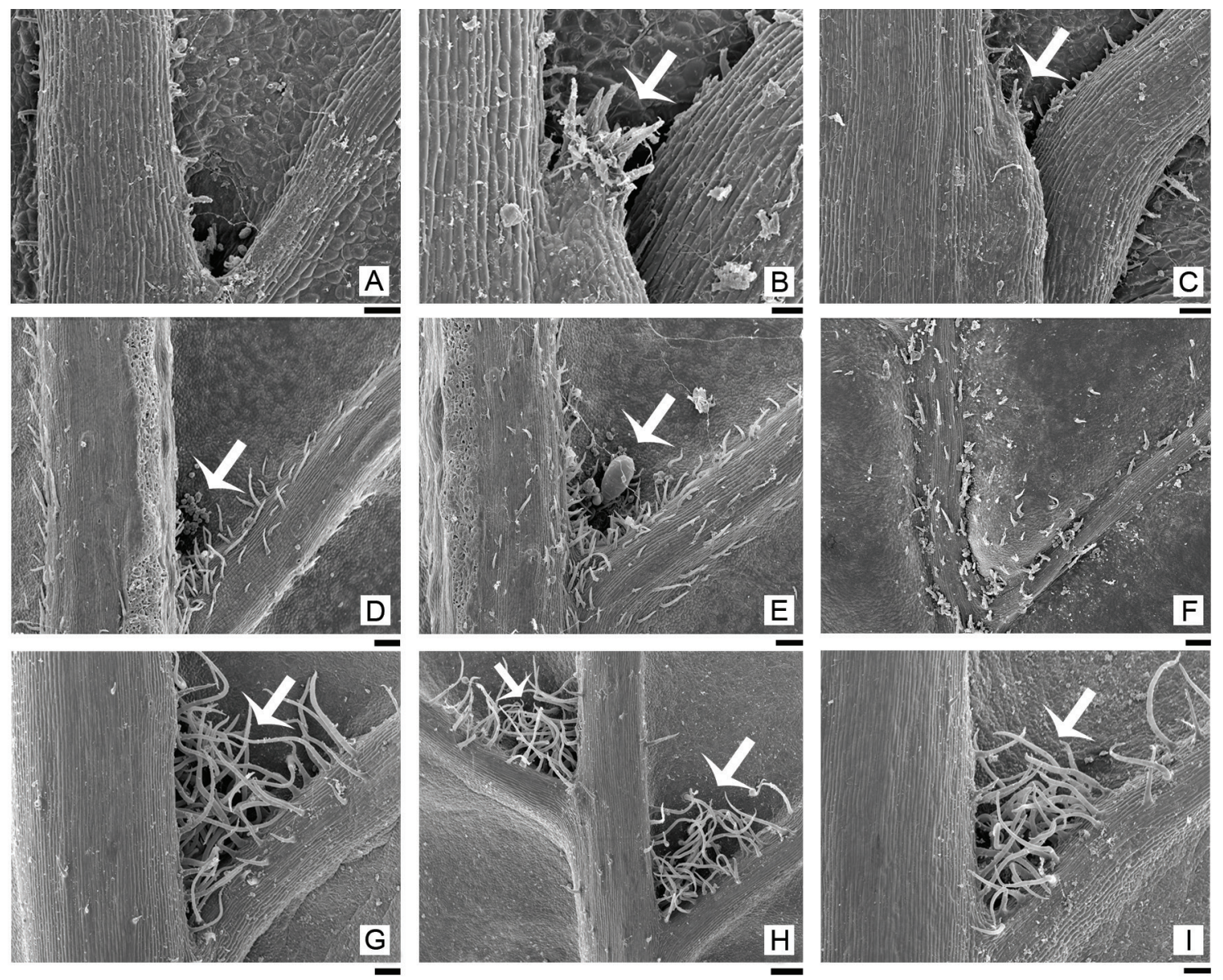

Fig. 3. Domacios en bolsillo. A-H: vista superficial con MEB. A-C: Psychotria carthagenensis, A: domacio glabro. B-C: domacio con tricomas uniseriados cortos rodeando la abertura (flecha). D-F: Machaonia brasiliensis, D: domacio glabro con rastros del ácaro (flecha). E: ácaro ingresando al domacio (flecha). F: domo del domacio en el haz. G-I: Coutarea hexandra: domacios con tricomas uniseriados largos rodeando la abertura (flechas). Escalas: A, C, D-G, I: $100 \mu \mathrm{m}$. B: $50 \mu \mathrm{m}$. H: $200 \mu \mathrm{m}$.

\section{Discusión y Conclusiones}

Importancia taxonómica a nivel tribal, genérico y especifico

A nivel de familia, la presencia de domacios es un carácter plesiomórfico debido a que aparece tempranamente en la familia. A nivel tribal, el domacio tiene nulo o escaso valor debido a que se puede observar la presencia de más de un tipo de domacio en las diferentes tribus. Con el fin de establecer la importancia filogenética de los datos morfológicos obtenidos, los mismos fueron contrastados con la clasificación de familia propuesta por Bremer \& Eriksson (2009). En base a esta clasificación, la familia se encuentra dividida en tres subfamilias: Rubioideae, Ixorideae y Cinchonoideae. En la Fig. 6 se encuentran representadas con asterisco (*) las tribus con representantes nativos en Argentina y a la derecha se observan los tipos de domacios presentes en cada grupo. En una primera instancia, se observa que los diferentes tipos de domacios analizados aparecen más de una vez y de manera independiente en las diferentes tribus de las tres subfamilias, lo cual nos permite afirmar que a nivel tribal este carácter no presenta utilidad para dividir grupos. En base al 
Bol. Soc. Argent. Bot. 50 (4) 2015
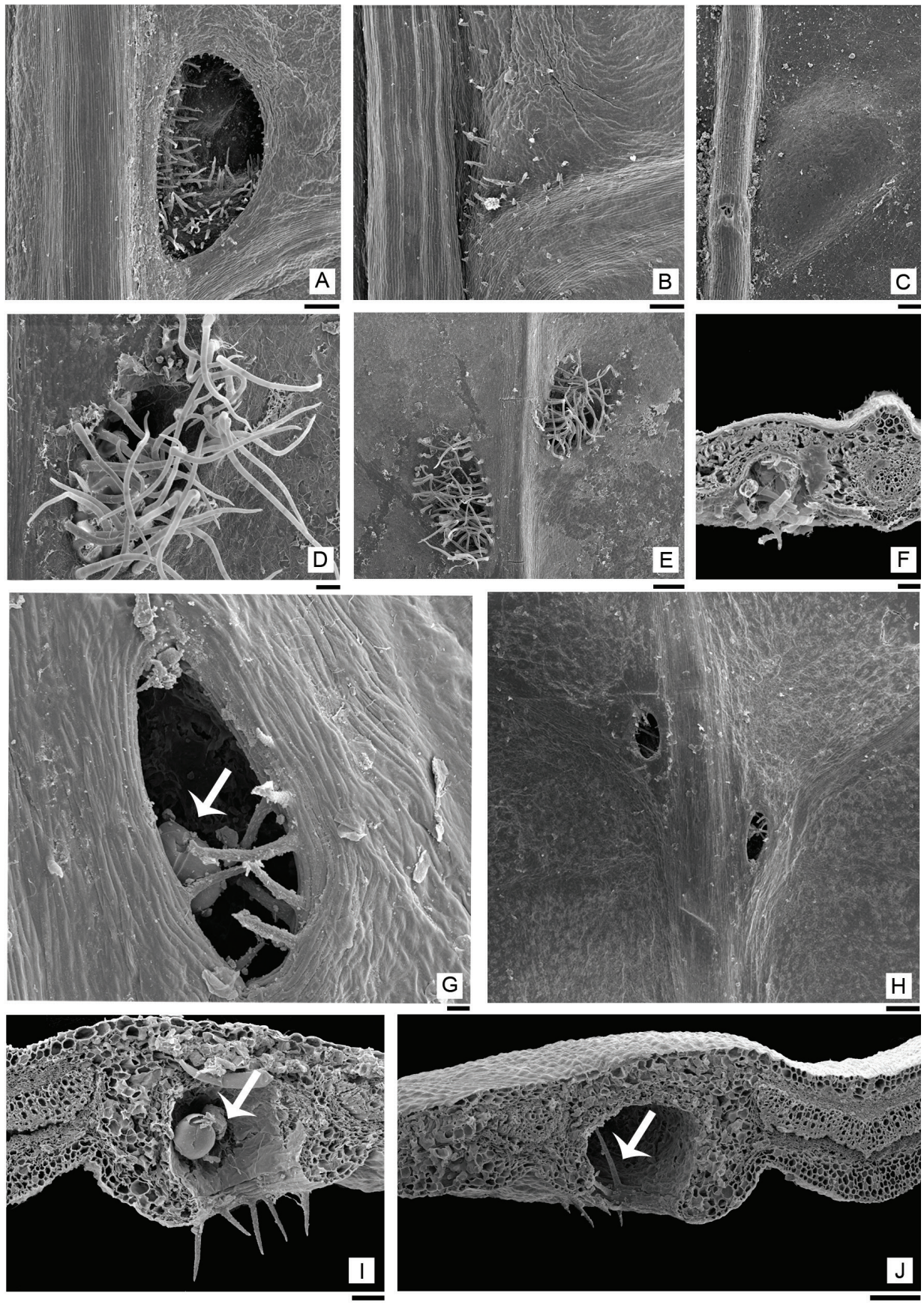

Fig. 4. Domacios en cripta. A-E: vista superficial con MEB, F, I-J: CT con MEB. A-B: Coussarea contracta, A: domacio con tricomas unicelulares cortos y largos en su interior. B: domo en el haz foliar, correspondiente al domacio del envés. C-F: Chomelia obtusa, C: domo en el haz foliar. D-E: tricomas uniseriados largos rodeando la abertura y en el interior del domacio. F: domacio con tricomas uniseriados. G-J: Manettia cordifolia, G: tricomas unicelulares cortos rodeando la abertura del domacio con ácaro en su interior (flecha). H: envés foliar con domacio localizado en la conexión entre la vena principal y las venas secundarias. I: domacio con huevo de ácaro en la cavidad (flecha). J: detalle de la cripta con tricomas unicelulares en el interior (flecha). Escalas: A-B, E, H: $200 \mu \mathrm{m}$. C, J: $100 \mu \mathrm{m}$. D, F, I: $50 \mu \mathrm{m}$. G: $20 \mu \mathrm{m}$. 

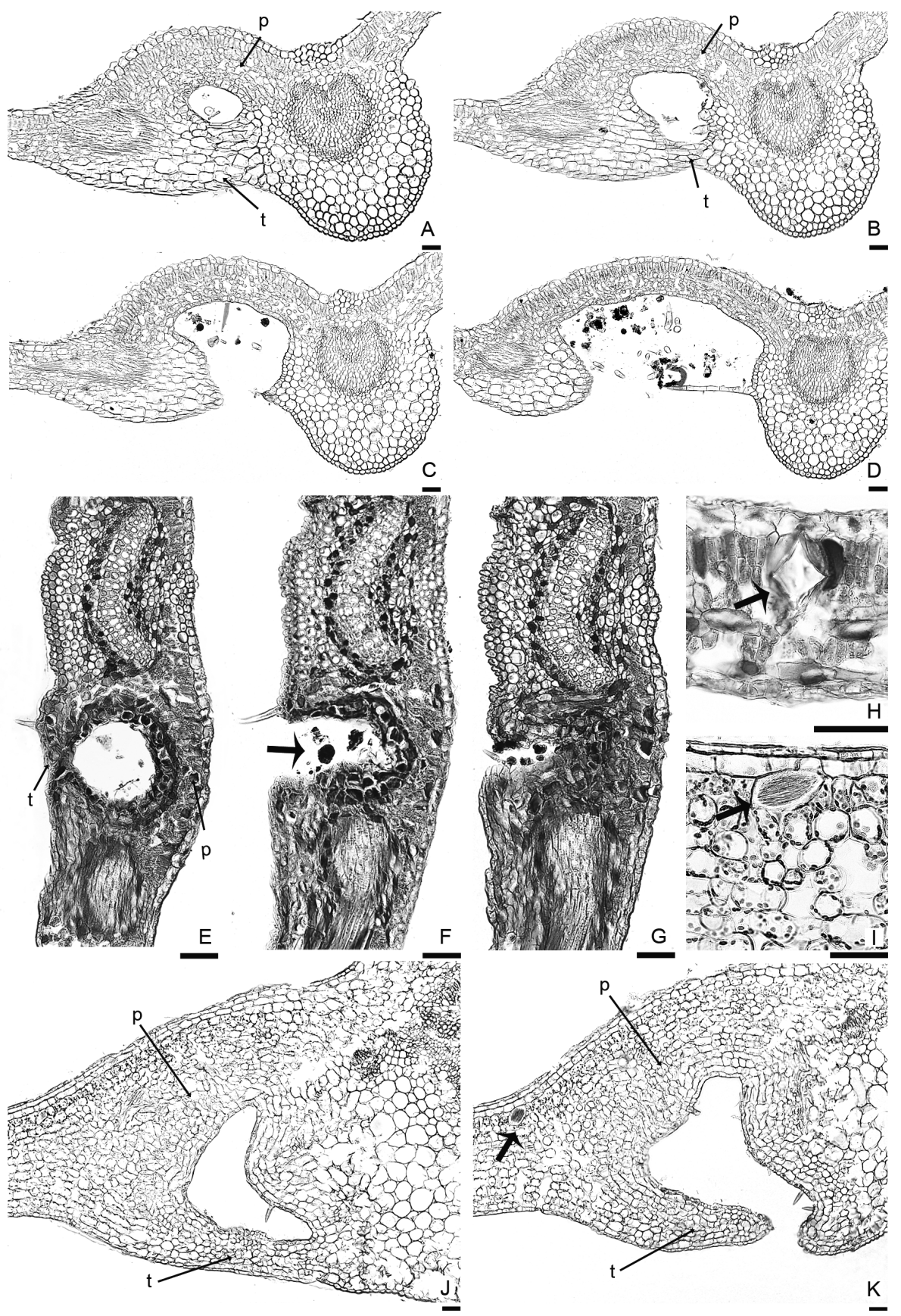

Fig. 5. Anatomía de domacios foliares en bolsillo y cripta en CT de lámina foliar (MO). A-D: domacio en bolsillo en Coutarea hexandra, A-B: secuencia de transcortes mostrando la cavidad con el piso ( $p$ ) y techo (t). C-D: cavidad del domacio abierta, con tricomas unicelulares cortos y uniseriados largos. E-H: Manettia cordifolia, E-G: secuencia de transcortes de la cavidad del domacio. E: estructura del domacio con piso (p) y techo (t). F: rastro de ácaros (flecha). G: cavidad del domacio cerrándose. H: detalle de idioblasto tanífero e idioblasto con cristal romboidal (flecha). I-K: Coussarea contracta, I: detalle de idioblasto con rafidios (flecha). J-K: estructura de la cripta con piso (p) y techo (t) con tricomas unicelulares cortos en el interior e idioblasto con rafidios (flecha). Escalas: A-K: $50 \mu \mathrm{m}$. 


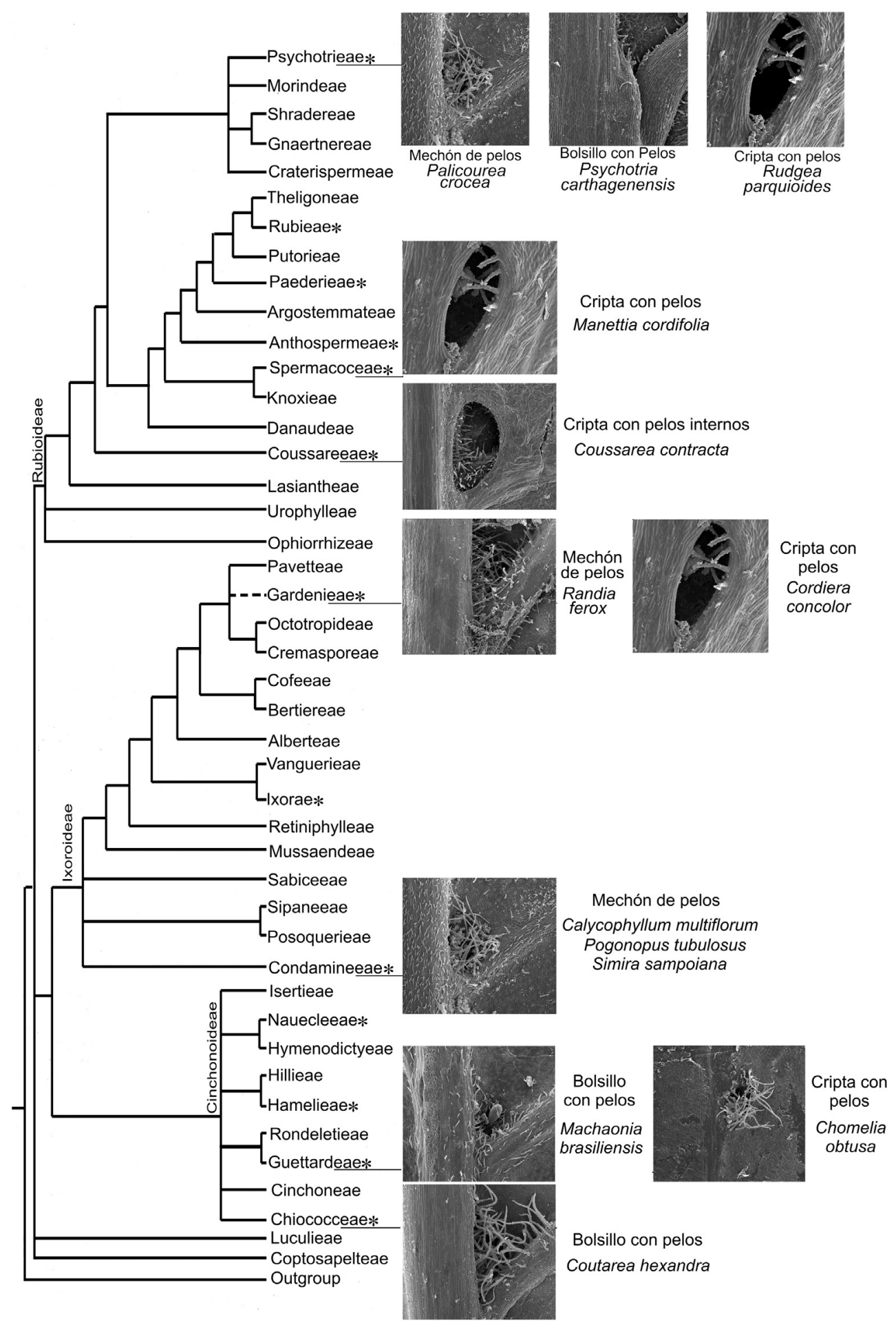

Fig. 6. Clasificación actual de la familia Rubiáceas, indicando las tres subfamilias; con $\left({ }^{*}\right)$ se marcan las tribus con representantes en Argentina y a la derecha los tipos de domacios encontrados en cada especie. Modificado de Bremer \& Eriksson, 2009. 
material analizado de Argentina, se observaron que las tribus Gardenieae, Guettardeae y Psychotrieae están representadas por más de un tipo de domacio.

A nivel específico adquieren importancia principalmente cuando son fácilmente identificables o bien cuando se compara ausencia o presencia (cripta en Coussarea contracta o bolsillo de Psychotria carthagenensis). Coussarea contracta se diferencia claramente de la especie más cercana $C$. platyphylla, porque ésta última carece de domacios. Mientras que Psychotria carthagenensis puede diferenciarse de todas las demás especies argentinas del género por ser el único taxón con domacios en bolsillo presente en todas las hojas observadas a campo. Se menciona por primera vez la presencia de domacios para la tribu Spermacoceae, taxón principalmente herbáceo. El hallazgo de domacios en cripta en Manettia carece de valor taxonómico, debido a que en sólo dos ejemplares de 150 analizados presentaron domacios. Estos datos, claramente cuestionan su uso extensivo en taxonomía, sobre todo porque es un sufrútice con ramas aéreas volubles y herbáceas, y no una liana o árbol donde normalmente se citan domacios. Sin embargo, se resalta su presencia debido a que resulta importante su estudio futuro a fin de responder que factores influyen en la presencia/ausencia.

El principal estudio de domacios en Rubiáceas fue realizado por Barros (1959, 1962), quién analizó numerosas especies, tanto americanas como africanas. A pesar del minucioso análisis, el trabajo de Barros (loc. cit.) es empíricamente irreproducible debido a que no detalló los materiales testigos y muchos de los nombres utilizados no existen en la literatura, más aún, en ocasiones listó algunas especies en dos géneros simultáneamente [ej. Spermacoce verticillata L. y Borreria verticillata (L.) G. Mey.; Borreria verbenoides Cham \& Schltdl. y Spermacoce verbenoides (Cham. \& Schltdl.) Niederl., entre otras]. El caso particular de $B$. verbenoides, actualmente se encuentra bajo Galianthe verbenoides (Cham. \& Schltdl.). Sin embargo, como fue demostrado por Cabral (2009) en el momento en el que Barros llevó a cabo sus estudios, este taxón constituía un complejo de al menos cinco especies diferentes (G. verbenoides, G. grandifolia E.L. Cabral, G. lanceifolia E.L. Cabral, G. matogrossiana E.L. Cabral y $G$. ramosa E.L. Cabral). Esto es comprobable en las identificaciones de herbario, debido a la gran cantidad de especímenes de estas especies identificados como $B$. verbenoides. Esta mención es importante debido a que en $G$. verbenoides, o en algunas de las otras especies, Barros mencionó presencia de domacios. Se analizaron numerosos materiales de todas las especies involucradas en este complejo y no se pudo confirmar la presencia de domacio. Teniendo en cuenta que al igual que Manettia, Galianthe también pertenece a la tribu Spermacoceae, la mención en el primer género constituye el único registro comprobable en la tribu. A pesar de la dificultad de reproducir estos resultados, muchos de los datos mencionados por Barros (1959, 1962) fueron reproducidos prácticamente sin alteraciones por Clifford \& Brouwer (1990). Este último trabajo es una de las recopilaciones más consultadas en cualquier abordaje de domacios.

Dificultad en el reconocimiento del domacio foliar

De acuerdo a nuestros resultados, el domacio foliar tipo mechón de pelos puede llegar a causar confusión a la hora de utilizarlo como carácter taxonómico. Por ejemplo, Guettarda uruguensis tiene las hojas notoriamente pubescentes, con tricomas especialmente abundantes sobre las venas, lo cual oculta o disimula la presencia del domacio en la zona de la axilas de las venas, también formado por entrecruzamiento de pelos con una densidad mayor que en el resto de la lámina.

En otros casos se observó que con un análisis superficial se describió un tipo de domacio, mientras que con un análisis anatómico se confirma otro tipo. Por ejemplo, Chomelia obtusa fue descrito como mechón de pelos (Barbosa, 2007; Delprete, 2010), mientras que nuestros resultados confirman una cripta con pelos. En Coutarea hexandra se menciona como aglomerado de de pelos (Barros, 1959); axilas pubescentes (Bacigalupo, 1993) у axilas barbadas (Delprete, 2010), mientras que en este trabajo se describe como un bolsillo con pelos. En Calycophyllum multiflorum, Bacigalupo (1993) describe como pelos hirsutos en las axilas de las venas, definida aquí como domacios en mechón de pelos. En Cephalanthus glabratus, Barros (1962) menciona la presencia de domacios en mechón de pelos. En base a observaciones a campo y abundante material de herbario no se pudo comprobar su presencia. 


\section{Agradecimientos}

Los autores agradecen a los curadores de los herbarios mencionados, a los coleccionistas del material utilizado para los estudios anatómicos, y a los revisores por los valiosos aportes realizados. Este trabajo es parte de los resultados del trabajo final de graduación para obtener el título de Licenciada en Ciencias Biológicas de la primera autora y fue realizado con un subsidio de la Universidad Nacional del Nordeste (PICTO 199/2011).

\section{Bibliografía}

BACIGALUPO, N. M. 1974. Rubiaceae. 6a: 3-50. In: Burkart, A. E. (ed.) Fl. Ilustr. Entre Ríos. Instituto Nacional de Tecnología Agropecuaria, Buenos Aires.

BACIGALUPO, N. M. 1993. Rubiaceae. In: Cabrera, A. L. Fl. Prov. Jujuy 13: 375-437.

BACIGALUPO, N. M. \& E. L. CABRAL. 2003. Simira sampaioana (Rubiaceae), nuevo registro para la flora Argentina. Darwiniana 41: 43-45.

BACIGALUPO, N. M., E. L. CABRAL \& C. M. TAYLOR. 2008. Rubiaceae, In: Zuloaga, F. O.; O. Morrone \& M. J. Belgrano, (eds.), Catálogo de las Plantas Vasculares del Cono Sur. Monogr. Syst. Bot. Mo. Bot. Gard. 107: 2871-2920.

BARBOSA, M. R. V. 2007. Chomelia. Fl. Fanerog. Estado São Paulo 5: 287-291. FAPESP, Sao Paulo, Brasil.

BARROS, A. M. A. 1959. Ocorrência das domacias na família Rubiaceae. An. Esc. Super. Agric. Luiz de Queiroz. 16: 311-370.

BARROS, A. M. A. 1961. Ocorréncia das domacias nas Angiospermas. An. Esc. Super. Agric. Luiz de Queiroz. 18: 113-130.

BARROS, A. M. A. 1962. Ocorrência das domacias nas Rubiáceas II. An. Esc. Sup. Agric. Luiz de Queiroz, Brasil. 19: 1-21.

BREMER, B. \& T. ERIKSSON. 2009. Time tree of Rubiaceae: phylogeny and dating the family, subfamilies, and tribes. Int. J. Plant Sci. 170:766793.

BROUWER, Y. M. \& H. T. CLIFFORD. 1990. An annotated list of domatia bearing species. Notes Jodrell Lab. 12: 1-33.

CABRAL, E. L. 2009. Revisión sinóptica de Galianthe subg. Galianthe (Rubiaceae-Spermacoceae), con una sección nueva. Ann. Mo. Bot. Gard. 96: 27-60.

DELPRETE, P. G. 1999. Rondeletieae (Rubiaceae)Part I (Rustia, Tresanthera, Condaminea, Picardaea, Pogonopus, Chimarrhis,
Dioicodendron, Molopanthera, Dolichodelphys, and Parachimarrhis). Fl. Neotrop. Monogr. 77: $1-226$.

DELPRETE, P. G., L. B. SMITH \& R. M. KLEIN. 2004. Rubiaceae. Fl. Il. Catarin. 1: 1-344. Herbario Rui Barbosa, Itajaí, Brasil.

DELPRETE, P. G. 2010. Rubiaceae. Fl. Estados Goiás e Tocantins 40: 1-1610. Goiania, Brasil.

GOMES, M. 2007. Coussarea. Flora. Fanerogâmica Estado São Paulo 5: 299-306. FAPESP, Sao Paulo, Brasil.

GONZALEZ, A. M. \& C. L. CRISTÓBAL. 1997. Anatomía y ontogenia de semillas de Helicteres lhostzkyana (Sterculiaceae). Bonplandia 9: 287-294.

GONZALEZ, A. M. 2011. Domacios y nectarios extraflorales en Bignoniáceas: componentes vegetales de una interacción mutualística. Bol. Soc. Argent. Bot. 46: 271-288.

JACOBS, M. 1966. On domatia - the viewpoints and some facts. P. K. Ned. Akad. C. Biol. 69: 275-316.

JOHANSEN, D. A. 1940. Plant microtechnique. McGraw-Hill Book Co. Inc. New York and London, pp. 511.

JUDKEVICH, M. D. 2013 (inéd.). Estudios biosistemáticos en especies argentinas de Randia (Rubiaceae). Trabajo Final de Graduación. pp. 50. Universidad Nacional del Nordeste, Corrientes, Argentina.

LUQUE, R., H. C. SOUSA \& J. E. KRAUS. 1996. Métodos de coloração de Roeser (1972) - modificado - e Kropp (1972) visando a substituição do azul de astra por azul de alcião $8 \mathrm{GS}$ ou $8 \mathrm{GX}$. Acta Bot. Bras. 10: 199-212.

MASCHWITZ, U., B. FIALA, Y. F. LEE, Y. K.CHEY \& F. L. TAN. 1989. New and little known myrmecophytic associations from Bornean rainforests. Malay. Nat. J. 43: 106- 115 .

MATTOS, C. H. C., A. PALlini, F. F. CHAVES \& C. GALBIATI. 2004. Domácias do cafeeiro beneficiam o ácaro predador Iphiseiodes zuluagai Denmark \& Muma (Acari: Phytoseiidae) Neotrop. Entomol. 33:57-63.

MATTOS, C. H. C., A. PALLINI, F. F.CHAVES, J. H. SCHOEREDER \& A. JANSSEN. 2006. Do domatia mediate mutualistic interactions between coffee plants and predatory mites?. Entomol. Expe. Appl. 118: 185-192.

MINEIRO, J. L. C., M. E. SATO, A. RAGA \& V. ARTHUR. 2008. Population dynamics of phytophagous and predaceous mites on coffee in Brazil, with emphasis on Brevipalpus phoenicis (Acari: Tenuipalpidae). Exp. Appl. Acarol. 44: 277291.

MORAES, T. M. S., C. F. BARROS, S. J. NETO, V. M. GOMES \& M. DA CUNHA 2009. Leaf blade 


\section{F. Romero et al. - Domacios foliares en Rubiáceas argentinas}

anatomy and ultrastructure of six Simira species (Rubiaceae) from the Atlantic Rain Forest, Brazil. Biocell 33: 155-165.

MORAES, T. M. S., G. R. RABELO, C. R. ALEXANDRINO, S. J. SILVA NETO \& M. DA CUNHA. 2011a. Comparative leaf anatomy and micromorphology of Psychotria species (Rubiaceae) from the Atlantic Rainforest. Acta Bot. Bras. 25: 178-190.

MORAES, T. M. S., C. F. BARROS, C. K. GOULART, F. C. MIGUENS \& M. DA CUNHA. 2011b. Domatia and leaf blade structure of Rudgea eugenioides (Rubiaceae). R. Bras. Bioci. 9: 25-32.

NAKAMURA, T., T. TANIGUSHI \& E. MAEDA. 2002. Leaf anatomy of Coffea arabica $\mathrm{L}$. with reference to domatia. Jpn. J. Crop. Sci. 61: 642-650.

O'DOWD, D. J. 1994. Mite association with the leaf domatia of coffee (Coffea arabica) in North Queensland, Australia. Bull. Entomol. Res. 84: 361366.

ROBBRECHT, E. 1979. The African genus Tricalysia A. Rich. (Rubiaceae - Coffeeae). 1. A revision of the species of subgenus Empogona. Bull. Jard. Bot. Natl. Belg. 49: 239-360.

ROBBRECHT, E. 1987. The African genus Tricalysia A. Rich. (Rubiaceae). 4. A revision of the species of section Tricalysia and section Rosea. Bull. Jard. Bot. Natl. Belg. 57: 39-208.
ROBBRECHT, E. 1988. Tropical Woody Rubiaceae. Opera Bot. Belg. 1: 1-271.

ROMERO, G. Q., R. D. DAUD, A. T. SALOMAO, L. F. MARTINS \& R. J. F. FERES. 2011. Mites and leaf domatia: no evidence of mutualism in Coffea arabica plants. Biota Neotrop. 11: 27-34.

TEPE, E. J., M. A., VINCENT \& L. E. WATSON. 2007. Stem diversity, cauline domatia, and the evolution of ant-plant associations in Piper Sect. Macrostachys (Piperaceae). Am. J. Bot. 94: 1-11.

VEGA, F. E., R. OCHOA, C. ASTORGA \& D. E. WALTER. 2007. Mites (Arachnida: Acari) inhabiting Coffee domatia: a short review and recent findings from Costa Rica. Int. J. Acarol. 33: 291-295.

WILKINSON, H. P. 1979. The plant surface (mainly leaf), part IV domatia. In: Metcalfe, C. R. \& L. Chalk (eds.) Anatomy of the Dicotyledons, 2nd ed., Vol. 1. pp. 132-140. Oxford: Clarendon Press.

Recibido el 26 de junio de 2015, aceptado el 5 de octubre de 2015. 


\section{ApÉNDICE}

\section{LISTA DE ESPECIES ARGENTINAS DE RUBIÁCEAS}

Todas las especies se encuentran en el Herbario C. L. Cristóbal (CTES), salvo se indique lo contrario.

Borreria brachystemonoides Cham. \& Schltdl.: Prov. Corrientes: Dpto. San Miguel, 12 Km NE de San Miguel, Ea. Curuzu Laurel, 2747’56”S, 57²7’21’W, 28-II-1990, Vanni 1510. Prov. Entre Ríos: Dpto. Federación, Sta. Ana, 3054’40”S, 5755’53”W, Burkart 21932 (SI). Prov. Misiones: Dpto. El Dorado, Ruta 17, a $28 \mathrm{Km}$ al E del cruce con Ruta 12, 20¹7'58’S, 54²5'40”W, 26-III-1996, Tressens 5566. Borreria brownii (Rusby) Standl. Prov. Jujuy: Dpto. Ledesma, Calilegua, camino a los Cafetales, 2342'50”S, 6442'14”W, 11-II-1980, Cabrera 31480. Borreria dasycephala (Cham. \& Schltdl.) Bacigalupo \& E.L. Cabral: Prov. Buenos Aires: Dpto. La Matanza, Tapiales, 3445’27'S, 58³7'19'W, 24-XII-1939, Hunziker 221 (CORD). Prov. Jujuy: Dpto. San Pedro, camino a la Mendieta, Ruta 56, 2415'43”S, 65²'16”W, 06-XII-2006, Salas 197. Prov. Misiones: Dpto. Gral. M. Belgrano, Bdo. de Irigoyen, Miguel 18. Prov. Salta: Dpto. Sta. Victoria, Los Toldos, 22¹9'60”S, 6443'11'W, 10-XII-2006, Salas 246. Borreria krapocarmeniana E.L. Cabral \& L.M. Miguel: Prov. Corrientes: Dpto. Ituzaingó, Ruta 14, cercano a policía caminera, Miguel 28. Prov. Misiones: Dpto. Candelaria, Sta. Ana, III-1945, Montes 1250 (CTES, LIL). Borreria loretiana E.L. Cabral: Prov. Misiones: Dpto. Candelaria, Loreto, 27¹9’S, 55²2’W, 19-XII-1981, Cabral 309. Borreria marticrovettiana E.L. Cabral: Prov. Misiones: Dpto. San Ignacio, Teyú Cuaré, 7 Km SW de San Ignacio, 27¹6’3”S, 55³3’28’W, 02-III-2004, Schinini 36453. Borreria nelidae E.L. Cabral: Prov. Misiones: Dpto. Concepción, 9 Km al NW de Concepción de la Sierra, 2759'S, 55³1'W, 11-III-1969, Krapovickas 15151. Borreria orientalis E.L. Cabral, L.M. Miguel \& R.M. Salas: Prov. Misiones: Dpto. Iguazú, Parque Nacional Iguazú, 2552’39”S, 54²4’40”W, 07-VIII1991, Vanni 2752. Borreria palustris (Cham. \& Schltdl.) Bacigalupo \& E.L. Cabral: Prov. Misiones: Dpto. San Pedro, Parque Provincial Moconá, costa del Río Uruguay, 279'S, 5353’W, 14-XI-1995, Guaglianone 2833. Borreria poaya (A. St.-Hil.) DC.: Prov. Misiones: Dpto. San Ignacio, Parque Provincial Teyú Cuaré, Sobrado 109. Borreria quadrifaria E.L. Cabral: Prov. Corrientes: Dpto. San Miguel, 12 Km NE de San Miguel, Ea. Curupayty, 2747'56”S, 57²7’21’W, 03-III-1990, Vanni 1695. Prov. Misiones: Dpto. Iguazú, Uruguaí, 2552’39’'S, 54²4’40”'W, 16-XII-1948, Schwarz 7004. Borreria spinosa Cham. \& Schltdl. Prov. Formosa: Dpto. Laishí, Reserva Ecológica El Bagual, 26²7’38'S, 58³4'26”W, 07-XI-1998, Di Giácomo 398. Prov. Jujuy: Dpto. M. Belgrano, 24²'55”S, 65²5’27”W, 07-XII-2007, Cabral 832. Prov. Salta: Dpto. Anta, Ruta Prov. 5, camino a las Lajitas, 2448’53”S, 6349’52”W, 17-XII-2006, Salas 320. Prov. San Luis: Dpto. Chacabuco, 0,5 Km al E del acceso a Papagayos, cerro en faldeo O de la Sierra de Comechingones, 32॰40’30”S, 6458'40”W, 14-I-2004, Pozner 213. Borreria sulcata (Bacigalupo) E.L. Cabral, Prov. Corrientes: Dpto. Santo Tomé, Río Aguapey y Ruta 14, 28¹2’29”S, 56¹3’41”W, Quarín 2616. Borreria schumannii (Standl. ex Bacigalupo) E.L. Cabral \& S.V. Sobrado: Prov. Corrientes: Dpto. Ituzaingó, Ruta 14, cercano a policía caminera, Miguel 27. Prov. Misiones: Dpto. Guaraní, Predio Guaraní, Ruta 15, frente a Tramo I, 26 54'59'S, 54¹2'18'W, 16-IX-1998, Tressens 6062. Borreria tenella (Kunth) Cham. \& Schltdl.: Prov. Misiones: Dpto. San Ignacio, acceso a Club del Río, 27¹6’32”S, 55³3’24”W, 28-II-2010,

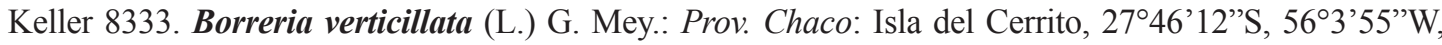
01-III-2006, Neiff 2132. Prov. Corrientes: Dpto. Santo Tomé, Ruta 68, 28¹2’29”'S, 56¹3’41’W, 11-XII2007, Cabaña 6. Prov. Misiones: Dpto. Apóstoles, $10 \mathrm{Km} O$ camino a Azara. Miguel 24. Prov. Corrientes: Dpto. San Roque, Ea. Caaguazú, 11 Km NE de Chavarría, camino a Tacuaritas, 28`43'34'S, 5840'32”W,

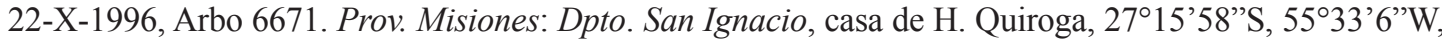
24-XI-2005, Zuloaga 8889 (CTES, SI). Prov. Entre Ríos: Dpto. La Paz, camino a Paraná, 3053'23”S, $59^{\circ} 32^{\prime} 40^{\prime}$ 'W, Burkart 21429 (SI).

Calycophyllum multiflorum Griseb.: Prov. Formosa: Dpto. Ingeniero Juárez, $3 \mathrm{Km}$ pasando el pueblo, 18-III-2014, Salas 497. Prov. Formosa: Ea. Guaycolec, Pilagá S.A., 2554’S, 58¹3’W, 25-VI-1999, Tressens 6311. Prov. Jujuy: Dpto. Sta. Bárbara, Aguas Calientes, 2347’15”S, 64²6’35”W, 10-II-1980, Cabrera 31435 (SI). 
Cephalanthus glabratus (Spreng.) K. Schum.: Prov. Corrientes: Dpto. Concepción, Ea. Yatay Corá, $50 \mathrm{Km}$ NE de Chavarría, 28²1’31”S, 5801'56”W, 23-X-1996, Arbo 6797. Prov. Entre Ríos: Dpto. Concordia, Est. Agronómica, 3122'’S, 587’W, I-1927, Burkart 979 (SI). Prov. Misiones: Dpto. San Pedro, Parque Provincial Moconá, costa del Río Uruguay, bajada al salto, $27^{\circ} 8^{\prime} \mathrm{S}, 53^{\circ} 53^{\prime} \mathrm{W}$, Seijo 849.

Chiococca alba (L.) Hitchc.: Prov. Corrientes: Dpto. Ituzaingó, Ruta 38 y Rio Aguapey, 2743’25”S, 564'0”'W, 12-II-1991, Tressens 3825. Prov. Misiones: Dpto. Candelaria, Ruta 12, $18 \mathrm{Km} \mathrm{NE} \mathrm{de}$ Candelaria, 27²6'22”S, 55³5’35”'W, 21-III-1997, Tressens 5736.

Chomelia obtusa Cham. \& Schltdl.: Prov. Corrientes: Dpto. Ituzaingó, Ea. Sta. Rita, 27³'S, 56²'W, 16-II-1991, Tressens 3950. Prov. Corrientes: Dpto. Santo Tomé, Ruta 40, camino a Colonia Pellegrini. 28`25’02,16’S, 56³2’33’W, 24-I-2014, Florentín 3. Prov. Misiones: Dpto. San Pedro, Parque Provincial Moconá, 07-III-2013, Judkevich 47. Prov. Misiones: Dpto. Guaraní, Predio Guaraní, Arroyo Soberbio, cerca del límite con IPS, 2654'59”S, 54¹8'0”W, 03-XI-1999, Tressens 6519.

Coccocypselum hasslerianum Chodat.: Prov. Misiones: Dpto. Guaraní, Predio Guaraní, 2654'59”S, 5412’18”W, 07-V-1997, Tressens 5839. Coccocypselum lanceolatum (Ruiz \& Pav.) Pers.: Prov. Misiones: Dpto. Guaraní, Predio Guaraní, 2654’59”S, 54¹2’18”W, 17-IX-1998, Tressens 6071. Prov. Salta: Dpto. Sta. Victoria, Los Toldos, 22¹9’13”S, 6443’0”W, 10-XII-2006, Salas 225.

Coccocypselum pulchellum Cham.: Prov. Misiones: Dpto. Guaraní, Predio Guaraní, Keller 3946.

Cordiera concolor (Cham.) Kuntze: Prov. Misiones: Dpto. San Ignacio, Teyú Cuaré, 27¹6’45,35”S, 55³4’30’'W, 01-III-2013, Judkevich 11.

Coussarea contracta (Walp.) Müll. Arg.: Prov. Misiones: Parque Provincial Cruce Caballero, Sendero Curi-y, 26³1'08”S, 5359’20,2”W, 05-III-2013, Judkevich 30. Coussarea platyphylla Müll. Arg.: Prov. Misiones: Dpto. Iguazú, Parque Nacional Iguazú, Islas del Paseo Superior, 2552’39”S, 54²4'40”W, 29-II-1996, Vanni 3534.

Coutarea hexandra (Jacq.) K. Schum.: Prov. Corrientes: Dpto. Ituzaingó, Ea. Sta. Rita, 27³'0”S, 56²'0”W, 16-II-1991, Tressens 3929. Prov. Jujuy: Dpto. Ledesma, Parque Nacional Calilegua,

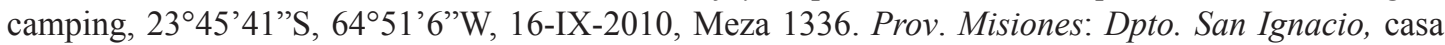

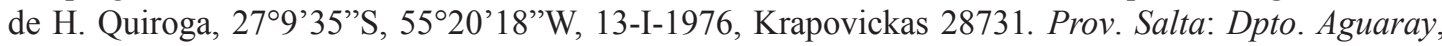
$20^{\circ} 20^{\prime} 47,4$ 'S, 6348'11'”, 10-XII-2012, Sato 201.

Cruckshanksia hymenodon Hook. \& Arn.: Prov. San Juan: Dpto. Calingasta, 01-III-1992, Kiesling 8051 (SI). Cruckshanksia macrantha Phil.: Prov. La Rioja: Dpto. Vinchina, 14-XII-1996, Biurrun 4557 (CORD). Cruckshanksia palmae Clos.: Prov. San Juan: Dpto. Calingasta, Río Melchor a Co. Guanaqueros, 06-II-1991, Kiesling 7826 (SI).

Deppea blumenaviensis (K. Schum.) Lorence.: Prov. Misiones: Dpto. Guaraní, Predio Guaraní, 2654'59”S, 54¹2'18”W, 02-IX-1999, Tressens 6404.

Diodia kuntzei K. Schum.: Prov. Corrientes: Dpto. Concepción, borde de laguna, Salas 322. Diodia saponariifolia (Cham. \& Schltdl.) K. Schum.: Prov. Misiones: Dpto. San Pedro, Parque Provincial Moconá, embarcadero Pedra Bugre, Salas 392.

Faramea cyanea Müll. Arg.: Prov. Misiones: Dpto. Iguazú, Parque Nacional Iguazú, 2552’39”S, 54²4’40”W, 27-III-1996, Tressens 5581.

Galianthe bisepala E.L. Cabral: Prov. Salta: Dpto. La Viña, Quebrada de las Conchas, entre la Salamanca y el Hongo, Ruta 68 Km, 25²3’21”S, 65³7'2”W, 10-III-1990, Novara 9630. Galianthe brasiliensis (Spreng.) E.L. Cabral \& Bacigalupo: Prov. Buenos Aires, Archipiélago Solís, Isla Solís, 15-XII-2003, Hurrell 5478 (SI). Prov. Misiones: Dpto. Libertador General San Martín, Aldea Guaraní Teko'a Miri, 2653'24”S, 557'38'W, 07-IV-2011, Keller 10064. Galianthe centranthoides (Cham. \& Schltdl.) E.L. Cabral: Prov. Misiones: Dpto. Candelaria, Bonpland, Arroyo Mártires Chico, 27²6’22”S, 55³5’35”W, 16-I-1976, Krapovickas 28791. Galianthe dichasia (Sucre \& C.G. Costa) E.L. Cabral: Prov. Misiones: Dpto. Gral. M. Belgrano, Ayo. Pepirí Guazú, 260’10”S, 5358’36”W, 23-I-1983, Guaglianone 1147. Galianthe equisetoides (Cham. \& Schltdl.) E.L. Cabral: Prov. Corrientes: Dpto. Ituzaingó, $11 \mathrm{Km} \mathrm{S}$ de Ruta 12, desvío a Gdor. Virasoro, 2743’25”S, 5644’0”W, 29-XI-1970, Krapovickas 16568. Galianthe eupatorioides (Cham. \& Schltdl.) E.L. Cabral: Prov. Corrientes: Dpto. Ituzaingó, Establecimiento 


\section{Bol. Soc. Argent. Bot. 50 (4) 2015}

Puerto Valle, SHELL C.A.P.S.A, 27³6’S, 56³0’0’W, 29-III-2000, Tressens 6600. Prov. Misiones: Dpto. San Ignacio, Parque Provincial Teyú Cuaré, 279’35”S, 55²0’18”W, 25-III-1996, Tressens 5558. Galianthe fastigiata. Griseb.: Prov. Corrientes: Dpto. Santo Tomé, Ruta 12 y Río Aguapey, 28`12’29”S, 56 13'41'W, 03-XI-2007, Ferrucci 2802. Prov. Misiones: Dpto. Candelaria, Loreto, 27²6’22”S, 55³5’35”,W, 19-XII-1981, Cabral 337. Galianthe hispidula (A. Rich. ex DC.) E.L. Cabral \& Bacigalupo: Prov. Misiones: Dpto. Guaraní, Predio Guaraní, 265'ㄱ, 54²12'W, 02-IX-1999, Tressens 6413. Galianthe laxa (Cham. \& Schltdl.) E.L. Cabral: Prov. Misiones: Dpto. Candelaria, alrededores de

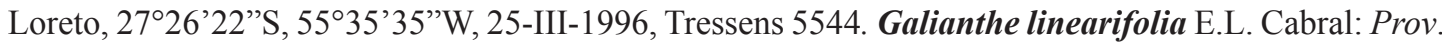
Misiones: Dpto. Apóstoles, Spegazzini 1 (BAB, LP). Galianthe montesii E.L. Cabral: Prov. Misiones: Dpto. M. Belgrano, San Antonio, 260'10”S, 5358'36”W, 02-X-1949, Montes 7036. Galianthe thalictroides (K. Schum.) E.L. Cabral: Prov. Corrientes: Dpto. Santo Tomé, Ayo. Chimiray y Ruta 40, 09-XII-1981, Tressens 1886. Prov. Misiones: Dpto. Candelaria, Cementerio de Bonpland, 27²8'46”S, 55'29'58”'W, 26-II-2011, Keller 9782. Galianthe valerianoides (Cham. \& Schltdl.) E.L. Cabral: Prov. Corrientes: Dpto. Santo Tomé, Ayo. Chimiray y Ruta 40, 09-XII-1981, Tressens 1885. Prov. Misiones: Dpto. Candelaria, Loreto, 27²6’22”S, 55³5’35”W, 12-III-1987, Fontana 259.

Galium antarcticum Hook. f.: Prov. Neuquén: Dpto. Huiliches, Parque Nacional Lanín, entre Junín de los Andes y Lago Lolog, 4000’4”S, 71 ${ }^{\circ} 20^{\prime} 0^{\prime}$ 'W, 1110 m, 22-I-2003, Cocucci 2416 (CORD, SI). Prov. Sta. Cruz: Río Coy, Hatcher s.n. (NY). Prov. Tierra del Fuego: Dpto. Ushuaia, Isla de los Estados y Canal de Beagle, VI-1902, Rodríguez 12025 (BAB, CTES). Islas Malvinas: West Point Island, Sladen 123 (BM). Galium aparine L.: Prov. Buenos Aires: Dpto. General Pueyrredón, Cerro La Peregrina, 3756'24'S, 5747’59”W, 05-X-2013, Giussani 565. (SI). Prov. Chubut: Dpto. Escalante, 4549’24”'S, 6758'18”W, 28-X-2005, Cocucci 3597 (SI). Prov. Río Negro: Dpto. Bariloche, Parque Nacional Nahuel Huapi,

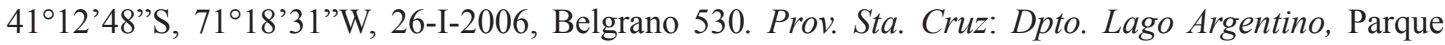
Nacional Los Glaciares, 50²9’14’S, 72॰57’30”W, 30-I-2003, Cocucci 2490 (SI). Prov. Tierra del Fuego: Dpto. Río Grande, Ea. Menendez Behety, 5345’S, 6757’ W, XII-1993, Giussani 299 (SI). Galium atherodes Spreng.: Prov. Misiones: Dpto. Capital, Molfino 189 (F). Prov. Sta. Fe: Pensiero 2005. Galium bigeminum Griseb.: Prov. Buenos Aires: Dpto. Coronel Suárez, Ea. El Lolén, 12-XII1979, Pertusi 97 (CTES, LP). Prov. Córdoba: Dpto. Cruz del Eje, cerca del Dique, 19-XII-1947, Meyer 13255 (CTES, LIL). Prov. Corrientes: Dpto. Monte Caseros, La Horqueta, 21-XII-1952, Nicora 6304 (CTES, SI). Prov. Entre Ríos: Dpto. Alcazarito, Lorentz 1537 (CORD, GOET). Prov. San Luis: Dpto. Ayacucho, Quilmes, 550 m, 4-XII-1944, Varela 724 (CTES, LIL). Galium comberi Dempster: Prov. Neuquén: Dpto. Huilches, Sta. Julia cerca de Colo Huincul, 1524 m, Comber 897 (K). Galium corymbosum Ruiz \& Pav.: Prov. Catamarca: Dpto. Ambato, Las Piedras Blancas, camino a Las JuntasHumaya, 1560 m, 3-IV-1995, Saravia Toledo 13393 (CTES, MSCN). Prov. Jujuy: Dpto. Sierra de Sta. Bárbara, Fries 267 (S). Prov. Salta: camino Cuesta del Arca-Trancas, Spegazzini 422 (F, LPS). Prov. Tucumán: Dpto. Tafí, RP 307, 15 Km al S de Tafí del Valle, 2658'02'S, 65³9'37’W, 1900 m, 05-III2004, Pozner 462 (CTES, SI). Galium equisetoides (Cham. \& Schltdl.) Standl.: Prov. Corrientes: Dpto. Paso de los Libres, 13-XI-1980, Ahumada 4122. Prov. Misiones: Dpto. Candelaria, Loreto, 12-II-1987, Fontana 249. Galium eriocarpum Bartl. ex DC.: Prov. Mendoza: Dpto. Malargüe, Mirador del Valle Hermoso, 3507’22”S, 70¹1'00”W, 2500 m, 28-I-2004, Prina 2417. Prov. Neuquén: Dpto. Minas, Cañada de Molina, 1260 m, 8-II-1996, Correa 9783 (BAB, CTES). Galium fuegianum Hook. f.: Prov. Chubut: Dpto. Tehuelches, Lago Vintter, 4410’56”S, 70³2’26”W, 19-I-1998, Nicora 10333 (SI). Prov. Neuquén: Dpto. Aluminé, arroyo frente al lago Aluminé, 2-II-1977, Crespo 2014 (BAB, CTES). Prov. Río Negro: Dpto. Bariloche, Valle del Chalhuaco, 14-I-1973, Agrasar 2985 (BAA, CTES). Prov. Sta. Cruz: Dpto. Lago Argentino, Cantino 136 (GH). Prov. Tierra del Fuego: Dpto. Harberton, Goodall 1113 (US). Galium gilliesii Hook. \& Arn.: Prov. San Juan: Dpto. Calingasta, Oeste de Barreal, El Pachon, Los Erizos, 09-II-1977, Kiesling 1385 (SI). Prov. Mendoza: Dpto. Las Heras, Laguna Los Horcones, ladera al W de la Vega, 3030 m, Hunziker 11179 (CTES. SI). Galium gracilicaule Ehrend. \& Bacigalupo: Prov. Jujuy: Dpto. Cochinoca, E de Abra Rechaite, 2254'12’'S, 66¹4’16”W, 4050 m, 17-III-1994, Taylor 11306 (MO, SI). Galium hirtum Lam.: Prov. Buenos Aires, Cerro La Peregrina, Eyerman 23686 (GH, UC). Prov. Corrientes: Dpto. Santo Tomé: Ea. Garrucho, Potrero Curuzú, 7-II-1972, Krapovickas 
21283. Prov. Entre Ríos: Dpto. Colón, Parque Nacional El Palmar, 1-IX-1982, Cusato 1541 (BAA, CTES). Prov. Misiones: Dpto. Concepción, Campos de San Lucas, Ea. La Angélica, 285'33”S, 55³2’43”W, 29-IX-2004, Múlgura 4052 (SI). Galium humile Cham. \& Schltdl.: Prov. Entre Ríos: Dpto. Federación, 24-IX-1961, Burkart 22763 (SI). Prov. Misiones: Dpto. Concepción, 29-IX-2004, Múlgura 4052 (MNES, SI). Galium hypocarpium (L.) Endl. ex Griseb.: Prov. Buenos Aires: Dpto. Zárate, Isla Botija, 3359'25"S, 598'15”W, 06-XI-2002, Ulibarri 1835 (SI). Prov. Chubut: Dpto. Futaleufú, PN Los Alerces, Río Los Arrayanes, 42³6’44”S, 7153’44”W, 26-III-2010, Olmstead 2010 (SI). Prov. Corrientes: Dpto. Concepción, camino desde "El Tránsito" hacia Yahaveré, Pirondo 109. Prov. Entre Ríos: Dpto. Islas del Ibicuy, Río Ceibo, 33³8’46”S, 5859'5”W, Burkart 4661 (SI). Prov. Misiones:

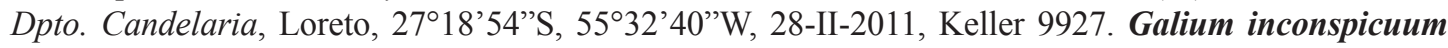
Phil.: Prov. Mendoza: Dpto. Malargüe, 20-XI-2001, Prina 1273 (SI). Prov. Neuquén: Dpto. Los Lagos, Parque Nacional Nahuel Huapi, travesía Punta Refugio, 1830 (SI, LIL). Galium latoramosum Clos: Prov. Corrientes: Dpto. Mercedes, Ea. Rincón del Diablo, 2844’S, 58²’W, 28-VIII-1998, Arbo 8006. Prov. Entre Ríos: Dpto. Colón, Parque Nacional El Palmar, patio de la intendencia. 31 ${ }^{\circ} 52^{\prime} 28^{\prime}$ 'S, 58 $12^{\prime}$ '36”W,

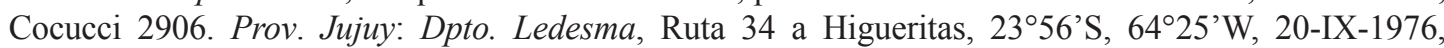
Cabrera 28027 (SI). Prov. Jujuy: Dpto. Ledesma, Parque Nacional Calilegua, Abra de Cañas (monolito), 2340’S, 6454’W, 03-XI-1998, Ahumada 8569 (SI). Prov. Misiones: Dpto. Guaraní, Predio Guaraní, 265'59”S, 5412’18”'W, 16-X-1997, Tressens 5963. Prov. Salta: Dpto. Sta. Victoria, de Lipeo a Los

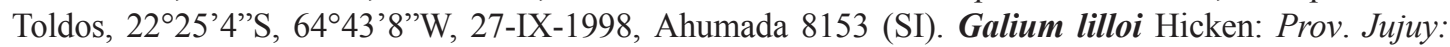
Dpto. Gral. M. Belgrano, RP 29 al Este de León, 240'S, 65²3'W, 19-XI-2002, Zuloaga 7845 (SI). Prov. Salta: Dpto. Quebradas del Río Toro y Río Blanco, I-1923, Vattuone 57 (SI). Prov. Tucumán: Dpto. Burruyacú, Las Tranquitas, 26³0'27'S, 6449'31"W, 09-XI-1900, Lillo 2560 (SI). Galium magellanicum Hook. f.: Prov. Chubut: Illin 469 (LP). Galium megapotamicum Spreng.: Prov. Misiones: Dpto. Candelaria, Loreto, RN 12, Keller 10835. Galium mollugo L.: Prov. Buenos Aires: Dpto. Capital, Stucker 22314 (CORD). Galium nigroramosum (Ehrend.) Dempster: Prov. Misiones: Dpto. Cainguás, Predio UNLP, Valle del arroyo Cuña Pirú, 276’49”S, 5448’43”W, 22-IX-1999, Biganzoli 568 (SI, CTES). Galium noxium (A. St.-Hil.) Dempster: Prov. Corrientes: Dpto. Saladas, San Lorenzo, 286'54'S, 58 46'4'W, 24-X-2007, Medina 22. Galium palustre L.: Prov. Buenos Aires: Dpto. Ensenada, Punta Lara, 3450’30”S, 5758'51'W, 08-XII-1967, Guaglianone s.n. (SI). Galium plumosum Rusby: Prov. Jujuy: Dpto. Valle Grande, Río Jordán y Ruta Prov. 83, 23³8'S, 6456’W, 05-XI-1998, Ahumada 8717 (SI). Prov. La Rioja: Dpto. Famatina, Flossdorf 3 (SI). Galium richardianum (Gillies ex Hook. \& Arn.) Endl. ex Walp.: Prov. Buenos Aires: Dpto. Isla Martín García, frente a la hostería. 27-III2007, Hurrell 6440 (SI). Prov. Chubut: Dpto. Sarmiento, RN 26, entre Sarmiento a Comodoro Rivadavia, 45³7'10”S, 6858'27’W, 17-XII-2011, Urtubey 590 (SI). Prov. Corrientes. Dpto. Mercedes, Río Corriente, $28^{\circ} 43^{\prime} 0^{\prime}$ S, 58 $8^{\circ}$ '0”W, 27-VIII-1998, Arbo 7952. Prov. Jujuy: Dpto. Tumbaya, camino de Purmamarca a Abra de Lipán, 2340'27’S, 65³6'15”'W, 12-II-2007, Zuloaga 9266 (SI). Prov. Misiones: Dpto. Guaraní, Predio Guaraní, 2654'59’'S, 54¹2’18”W, 31-VIII-1999, Tressens 6357. Prov. Neuquén: Dpto. Collón Curá, a casi 3 Km de Piedra del Aguila, 3959'3”S, 70²’31'W, 18-X-2005, Cocucci 3476. Prov. Salta: Dpto. Cachi, Cuesta del Obispo, La Cochera, 25¹0’12”S, 6551'14”W, 22-I-2002, Solís Neffa 854. Prov. San Luis: Dpto. Libertador General San Martín, El Parador, 32¹9’24”S, 65²4’5”W, 15-III-2011, Deginani 2138 (SI). Prov. Río Negro: Dpto. Bariloche, Cerro de la Buitrera, 42²5'35"S, 71³2'51'W, II-2012, Urtubey 737 (SI). Galium saxatile DC.: Prov. Tierra del Fuego, Moore 648 (LP). Galium suffructicosum Hook. \& Arn.: Prov. Mendoza: Dpto. Las Heras, Moseley 1 (BM). Galium tricornutum Dandy.: Prov. Chaco, 1931, Schulz 503. Galium uruguayense Bacigalupo.: Prov. Corrientes, Tressens 2893. Galium verum L.: Prov. Río Negro: Dpto. Adolfo Alsina, Meyer 7153 (LIL). Galium vile (Cham. \& Schltdl.) Dempster: Prov. Misiones: Dpto. Capital, Arroyo Itaembé, $27^{\circ} 28^{\prime} 53^{\prime \prime S}$, 5555'8”W, 28-VIII-1993, Guillén 276 (SI).

Genipa americana L.: Prov. Corrientes: Dpto. Mburucuyá, Ea. Sta. Teresa, 2759’27’S, 58¹0’58”W, 24-VIII-1976, Krapovickas 29593. Prov. Formosa, Ea. Guaycolec, Pilagá S.A., 2554'0”S, 58¹3'0”'S, 26-VI-1999, Tressens 6325. Prov. Misiones: Dpto. El Dorado, Ciudad de El Dorado, 26¹7'58”S, $54^{\circ} 25^{\prime} 40^{\prime \prime} \mathrm{W}$, Keller 323 . 
Geophila macropoda (Ruiz \& Pav.) DC.: Prov. Misiones: Dpto. Iguazú, Ruta 12, límite del Parque Nacional Iguazú, 2552’39’'S, 54²4’40”W, 10-I-1970, Krapovickas 15611. Geophila repens (L.) I.M. Johnst.: Prov. Corrientes: Dpto. Ituzaingó, Establecimiento Puerto Valle, 2741'S, 56²9' W, 28-III2000, Tressens 6545. Prov. Misiones: Dpto. Gral. M. Belgrano, Reserva Estricta San Antonio, $26^{\circ} 1^{\prime} 0^{\prime \prime S}$, 534''”, 12-III-2002, Múlgura 3266.

Guettarda uruguensis Cham. \& Schltdl:: Prov. Buenos Aires, Isla Martín García, 08-XI-2006, Hurrell 6484 (SI). Prov. Corrientes: Dpto. Mercedes, Ea. Yacaré, 2845'0”'S, 58³’0”W, 02-XII-1998, Arbo 8198. Prov. Corrientes: Dpto. Santo Tomé, Ruta 40, camino a Colonia Pellegrini, 28²5'02,16”S 56³2’33”W, 24-I-2014, Florentín 1. Prov. Entre Ríos. Dpto. Colón, Parque Nacional El Palmar, 3152’29”S, 58¹2’36”'W, Cocucci 2928. Prov. Misiones: Dpto. Montecarlo, próximo a RP 16, 26³8'31'S, 5434'57’'W, 28-XI-1999, Keller 122.

Hamelia patens Jacq.: Prov. Misiones: Dpto. Iguazú, Pto. Península, 2552’39”S, 5424’40”W, 02III-1996, Vanni 3575. Prov. Jujuy: Dpto. Ledesma, Calilegua, 2342’20”S, 6443’42”W, 09-II1980, Cabrera 31332 (SI). Prov. Salta: Dpto. Orán, Finca San Andrés, “Los Naranjos” 23²1'55”S, 6413'2”'W, 28-X-1997, Schinini 32933.

Heterophyllaea pustulata Hook. f.: Prov. Jujuy: Dpto. Sta. Bárbara, 24¹7'53”S, 64²9'5”'W, 19-I-2002, Solís Neffa 809. Prov. Tucumán: Dpto. Burruyacú, camino a Sierra de Medina, 26²6’48”S, 65¹'54”W, 15-XII-2008, Slanis 15 (SI).

Hoffmannia australis Lillo.: Prov. Jujuy. Dpto. Ledesma, PN Calilegua, Iudica 255 (SI). Prov. Salta: Dpto. Orán, Quebrada El Arrayasal, Legname 5796 C (LIL). Prov. Tucumán: Dpto. Chicligasta, Puesto Sta. Rosa, 28-II-1950, Meyer 15481 (CTES, LIL, SI). Hoffmannia peckii K. Schum.: Prov. Misiones: Dpto. San Pedro, Parque Provincial Moconá, sendero hacia la gruta, 14-V-2008, Salas 374 (CTES, SI).

Ixora venulosa Benth.: Prov. Misiones. Dpto. Iguazú, PN Iguazú, 2552’39”S, 54²4’40”W, 15-X-1993, Tressens 4533.

Leptostigma arnottianum Walp.: Prov. Río Negro: Dpto. Bariloche, entre Puerto Moreno y Pichi Mahuida,

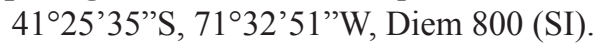

Machaonia brasiliensis (Humb.) Cham. \& Schltdl.: Prov. Chaco: Dpto. San Fernando, Barranqueras, Paranacito, 2741'56”S, 597'35”W, 05-IV-2007, Rotta 451. Prov. Corrientes: Dpto. Capital, 27²9'53”S, 5846’3”W, 12-II-1999, Franceschini 29. Prov. Misiones: Dpto. Azara, 2807’13,9”S, 55 7’19,9'W, 28-II-2013, Judkevich 9.

Manettia cordifolia Mart.: Prov. Corrientes: Dpto. Ituzaingó, Ea. Sta. Rita, 27³'0”S, 56 4'0”'W, 15-II1991, Tressens 3910. Prov. Jujuy: Dpto. Sta. Bárbara, camino del fuerte, 24¹'41”S, 64²4'55”W, 03-V-1999, Krapovickas 47687. Prov. Misiones: Dpto. Azara, 2802'45,4”'S, 55³9’30,3”W, Sobrado 133. Prov. Salta: Dpto. Candelaria, camino de El Jardín a Pampa Grande, 264’32”S, 65²9’4”W, 28 III-2009, Slanis 306 (SI). Manettia jorgensenii Standl.: Prov. Jujuy: Dpto. Gral. M. Belgrano, Abra de Salvear, 26²'47’'S, 65²4'49”'W, 24-III-1993, Deginani 123 (SI). Prov. Tucumán: Dpto. Chicligasta, Las Pavas, 27¹4'3”S, 6554'7'W, 01-XII-1915, Jörgensen 1908 (SI). Manettia paraguarensis Chodat: Prov. Misiones: Dpto. Guaraní, Predio Guaraní, 2654'59’'S, 54¹2’18”W, 18-II-1994, Tressens 1507.

Mitracarpus brasiliensis M.L. Porto \& Waechter: Prov. Misiones: Dpto. Guaraní, Predio Guaraní, camino al ayo. Soberbio, 265'59'S, 54²'18’'W, 17-IX-1998, Tressens 6070. Mitracarpus brevis K. Schum. \& R.E. Fr.: Prov. Jujuy: Dpto. Humahuaca, Empalme a Iturbe, 230'0”'S, 65²2'0”W, 20-I-1976, Cabrera 27390 (SI). Prov. Salta: Dpto. Cachi, Cuesta del Obispo, 259'47’S, 6550’27’W, 22-I-2002, Solís Neffa 876. Mitracarpus hirtus (L.) DC. Prov. Misiones: Dpto. Gral. M. Belgrano, $4 \mathrm{Km} \mathrm{NW}$ de San Antonio, 260'10’'S, 5358’36”'W, 29-III-1996, Tressens 5595. Prov. Salta: Dpto. Sta. Victoria, Los Toldos, camino al Parque Nacional Baritú, 22 19 '51'S, 6443'19”'W, 04-XII-2007, Cabral 812. Mitracarpus megapotamicus

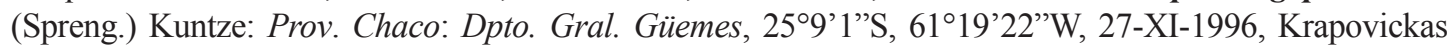
46859. Prov. Corrientes: Dpto. San Roque, Ea. Caaguazú, 2843’34”S, 5840’32”W, 22-X-1996, Arbo 6699. Prov. Entre Ríos: Dpto. La Paz, Ea. “La Esperanza”, 3053’23”S, 59³2’40”W, Burkart 21427 (SI). Prov. Formosa: Dpto. Laishí, Reserva Ecológica El Bagual, 26²7’38’S, 58³4’26”W, 15-V-1999, Di

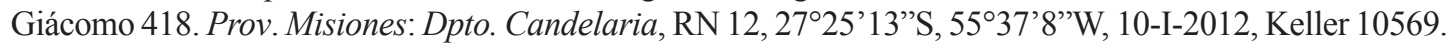
Prov. Salta: Dpto. Anta, RP 5, camino a las Lajitas, 2448'53”S, 6349'52”W, 17-XII-2006, Salas 321. 
Nertera granadensis (Mutis ex L. f.) Druce: Prov. Río Negro: Dpto. Near Laguna Frias, PN Nahuel Huapi, 23-II-1952, Pedersen 1580. Prov. Chubut: Dpto. Futaleufú, Lago Menendez, Aleszal Brazo Sur, 10-II1949, Soriano 3486 (MO).

Oldenlandia salzmannii (DC.) Benth. \& Hook.: Prov. Corrientes: Dpto. Mercedes, Ea. Rincón del

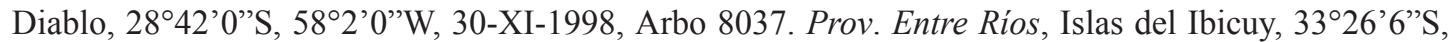
594’4”W, XII-1930, Burkart 3558 (SI). Prov. Misiones: Dpto. Concepción, Ea. de Elsa Prates sobre el Río Uruguay, 28`5'50”'S, 55³2’30”W, 29-I-2004. Biganzoli 1710 (SI). Prov. Neuquén: Dpto. Aluminé, Pulmarí, 397'2”S, 71¹'30”'W, 08-XII-1981, Cabrera 32957 (SI).

Oreopolus glacialis (Poepp.) Ricardi: Prov. Chubut: Dpto. Futaleufú, Chubut, entre Tecka y Corcovado,

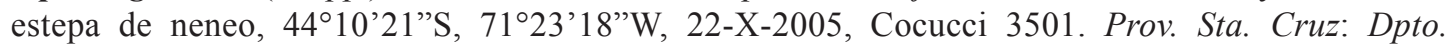
Magallanes, 30 Km W de San Julián, 4851'21’'S, 68²3'38”W, 05-XI-1997, Lutz 373.

Paederia brasiliensis (Hook. f.) Puff: Prov. Salta: Dpto. Orán, RP 18, a 3-4 Km del Puente Internacional, $22^{\circ} 43^{\prime} \mathrm{S}, 64^{\circ} 43^{\prime} \mathrm{W}, 1-\mathrm{V}-2003$, Morrone 4527. Prov. Jujuy: Dpto. Ledesma, Río Zora, margen izquierda, 21-VI-1998, Novara 11086 (MCNS).

Palicourea crocea (Sw.) Schult.: Prov. Corrientes: Dpto. Santo Tomé, Laguna Luna, 28`3'62”S, 56 45'72'W, 19-I-2000, Arbo 8575. Palicourea macrobotrys (Ruiz \& Pav.) DC.: Prov. Misiones: Dpto. Iguazú, PN Iguazú, Isla, 2552’39”S, 54²4’40”W, 30-III-1995, Vanni 3354.

Pogonopus tubulosus (A. Rich.) K. Schum.: Prov. Jujuy: Dpto. Ledesma, PN Calilegua, sendero "La

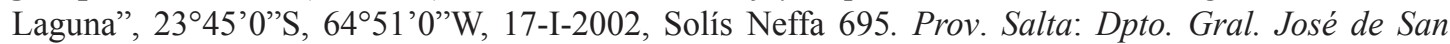
Martín, Jurisdicción Aguaray, 22¹6’60”S, 6350’60”W, 17-XII-2006, Salas 304.

Psychotria argentinensis Bacigalupo: Prov. Jujuy: Dpto. Ledesma, PN Calilegua, RP 83, Abra de Cañas a Mesada de las Colmenas, 2341'0”'S, 6454'54”W, 11-XI-2002, Zuloaga 7498 (SI). Prov. Salta: Dpto. Orán, Finca San Andrés, 235’32”S, 6447’11”W, 02-XI-1997, Schinini 33129. Psychotria brevicollis Müll. Arg.: Prov. Misiones: Dpto. San Pedro, PP Moconá, 278'0”S, 5353'0”W, Seijo 582. Psychotria carthagenensis Jacq.: Prov. Corrientes: Dpto. Concepción, Ea. Yatay Corá, $50 \mathrm{Km} \mathrm{NE}$ de Chavarría, 28²1’31”S, 58 1'56”W, 23-X-1996, Arbo 6801. Prov. Corrientes: Dpto. Santo Tomé, 28²5’02,16”S, 56²’33”W, 64 m. 24-I-2014, Florentín 6. Prov. Formosa: Dpto. Capital, Ea. Guaycolec, Pilagá S.A., 2554'0”S, 58 13'0”'W, 25-VI-1999, Tressens 6312. Prov. Jujuy: Dpto. Ledesma, PN Calilegua, 2341'39"S, 6451'52”W, 16-IX-2010, Meza Torres 1358. Prov. Misiones: Dpto. San Pedro, Parque Provincial Moconá. 27099'17’'S, 5353'26,2”W, 536 m. 06-III-2013, Sobrado 171. Prov. Misiones: Dpto. Guaraní, camino al Arroyo Soberbio, 2654'S, 54²12'W, 01-IX-1999, Tressens 6382. Prov. Salta: Dpto. Orán, Finca San Andrés, Casavindo, Río San Andrés, 23²1'55”S, 64¹3'2”W, 29-X-1997, Schinini 32951. Prov. Tucumán: Dpto. Monteros, Quebrada de los Sosas, 276’22”S, 65³7’55W, 27-IV-1992, Fortunato 3515. Psychotria deflexa DC.: Prov. Misiones: Dpto. Candelaria, 21-IV-1958, Montes 27374 (SI). Psychotria leiocarpa Cham. \& Schltdl.: Prov. Corrientes: Dpto. Santo Tomé, Ea. San Benito, 22 Km NW de Santo Tomé, 2812’29”'S, 56¹3'41”W, 10-IV-1992, Tressens 4075. Prov. Misiones: Dpto. San

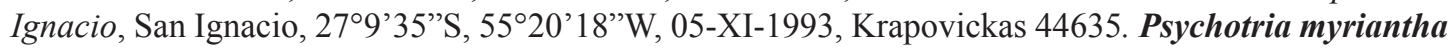
Müll. Arg.: Prov. Misiones: Dpto. San Pedro, 07-V-1999, Deginani 1084. Psychotria nitidula Cham. \& Schltdl.: Prov. Misiones: Dpto. Iguazú, PN Iguazú, 2552’39”S, 54²4’40”W, 07-VIII-1991, Vanni 2722. Psychotria suterella Müll. Arg.: Prov. Misiones: Dpto. Gral. M. Belgrano, al S de Bernardo de Irigoyen, 260'10"S, 5358'36”'W, 15-XII-1987, Eskuche 3085.

Randia ferox (Cham. \& Schltdl.) DC.: Prov. Corrientes: Dpto. Ituzaingó, $17 \mathrm{Km}$ NW de San Carlos, Ea. Rincón Chico, 2743’25”S, 5644’0”W, 14-II-1991, Tressens 3909. Prov. Misiones: Dpto. San Pedro, Parque Provincial Moconá, Mirador del Mirador del Yaboty, 2709’03,67’S, 5359'51,09”W, 07-III2013, Judkevich 34. Randia micracantha (Lillo) Bacigalupo: Prov. Jujuy: Dpto. San Pedro, RP 56, Cuesta de las Lajitas, 10 Km SW de La Mendieta, 24¹7'50’S, 6450’24”W, 13-V-1998, Krapovickas 47412.

Richardia brasiliensis Gomes: Prov. Corrientes: Dpto. Bella Vista, Colonia 3 de Abril, $5 \mathrm{Km}$ al N de Bella Vista, 28²7’31”S, 5856’4”W, 08-VIII-1998, Salgado 25. Prov. Formosa: Dpto. Laishí, Reserva Ecológica El Bagual, 26²7’38”S, 58³4’26”W, 15-X-1999, Di Giácomo 445. Prov. Misiones: Dpto. Iguazú, entrada a Puerto Esperanza y Ruta 12, 2552’39”S, 54²4’40”W, 11-XI-1996, Vanni 3773. Prov. 
Salta: Dpto. Rivadavia, Ruta 81, Km 1692, 234', 62²4’W, 15-I-2002, Solís Neffa 601. Richardia coldenioides Rusby: Prov. Córdoba: Dpto. Calamuchita, Río Tercero, 32¹1'4”S, 64³7'1”W, 09-I1940, Burkart 10443. (SI). Richardia grandiflora (Cham. \& Schltdl.) Steud.: Prov. Corrientes: Dpto. San Miguel, Ea. Poriajhu, 2747’56”S, 57²7’21”W, 06-XII-1992, Tressens 4261. Prov. Misiones:

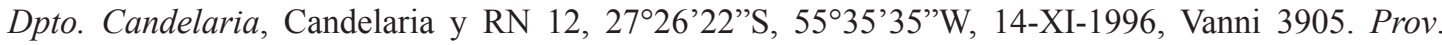
Salta: Dpto. José de San Martín, Ruta 34 Km 1374, cruzando el puente sobre el Río Seco, margen S, 231'47'S, 6351'11'W, 16-I-2002, Solís Neffa 677. Richardia humistrata (Cham. \& Schltdl.) Steud.: Prov. Misiones: Dpto. Gral. M. Belgrano, Bernardo de Irigoyen, Miguel 17. Richardia stellaris (Cham. \& Schltdl.) Steud.: Prov. Corrientes: Dpto. Itatí, Ramada Paso, 27¹8'21’S, 58²’27’W, 11-XII-2007, Cabaña Fader 1. Prov. Jujuy: Dpto. Gral. M. Belgrano, 24²'55'S, 65²5'27'W, 07-XII-2007, Cabral 831. Prov. Salta: Dpto. Sta. Victoria, Los Toldos, 22¹9'60”S, 6443’11”W, 10-XII-2006, Salas 245.

Rubia tinctorum L. Prov. Mendoza: Dpto. Guaymallén. Covas 239 (SI).

Rudgea parquioides (Cham.) Müll. Arg.: Prov. Misiones: Dpto. Guaraní, Predio Guaraní, 254'59”S, 54¹2'18'W, 14-X-1997, Tressens 5925.

Scherardia arvensis L.: Prov. Buenos Aires, Burkart 4581.

Simira sampoiana (Standl.) Steyerm.: Prov. Misiones: Dpto. Iguazú, PN Iguazú, Vanni 3413.

Spermacoce eryngioides Cham. \& Schltdl.: Prov. Chaco: Dpto. Gral Güemes, Paraje Costa Rica, Proyecto Reserva Ecológica APROMA, 5 Km E de 4 de Febrero, 259'1'S, 61¹9'22”W, 27-XI-1996, Krapovickas 46866. Prov. Córdoba: Dpto Calamuchita, Villa Rumipal, 32¹1'4”S, 64³7'1'W, 17-I1951, De la Sota 4019. Prov. Formosa: Dpto. Laishí, Reserva Ecológica El Bagual, San Francisco de Laishi, 26²7’38’S, 58³4’26”W, 30-IX-1998, Di Giácomo 389. Prov. Misiones: Dpto. Candelaria, RN 12, ca. de Est. de peaje, $27^{\circ} 25^{\prime} 23^{\prime}$ 'S, 55 37'8’W, 10-I-2012, Keller 10567. Prov. Tucumán: Dpto. Cruz Alta, Ruta Prov. 303, 4 Km al W de Las Cejas, 2655’5”S, 6459'37’W, 07-III-2004, Pozner 494 (CTES, SI). Prov. Salta: Dpto. Gral. José de San Martín, Aguaray, camino a Acambuco, Km 40, 229'35'S, 6354'60”W, 17-XII-2006, Salas 303. Spermacoce prostrata Aubl.: Prov. Entre Ríos, Islas del Ibicuy, Delta Entrerriano, Islas Orero, Mandel, 334ㄱ'S, 58 $45^{\prime}$ W, III-1937, Burkart 8366 (SI). Prov. Formosa: Dpto. Patiño, El Cogoik, 2450'17’S, 5959’39”W, 19-II-2004, Tressens 6927 (CTES, SI). Prov. Misiones: Dpto. Candelaria, Ruta 12 y Ayo. Yabebiry, 27²6’22”S, 55³5’35”W, 17-XII-1983, Cabral 415. Spermacoce riparia Cham. \& Schltdl.: Prov. Corrientes, Isla del curso del Río Paraná, $27^{\circ} 26^{\prime} 37^{\prime \prime}$, 584'44”'W, 12-XII-2005, Neiff 2110. Prov. Misiones: Dpto. Montecarlo, Puerto Piray, Miguel 13. Spermacoce tenuior L.: Prov. Jujuy: Dpto. San Pedro, Ruta Prov. 56, 3 Km de La Mendieta camino a S.S. de Jujuy, 24²0'S, 6459'W, 19-II-1998, Morrone 2777 (SI). Prov. La Rioja: Dpto. Gral. Belgrano, 16-III-2000, Biurrun 6427 (SI).

Staelia juarezii E.L. Cabral \& R.M. Salas: Prov. Formosa: Dpto. Ingeniero Juárez, Burkart s.n. Staelia thymoides Cham. \& Schltdl.: Prov. Entre Ríos: Dpto. Colón, Parque Nacional El Palmar, patio de la intendencia, 3152’29”S, 58¹2’36”W, Cocucci 2933. Prov. Misiones: Dpto. San Ignacio, Teyú Cuaré,

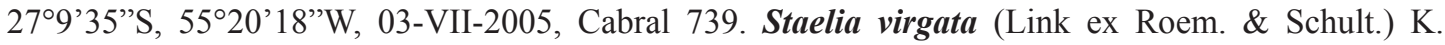
Schum.: Prov. Chaco: Dpto. Gral. Güemes, Nueva Pompeya, 259'1”S, 61¹9’22”W, 13-V-1970, Schulz 17425. Prov. Jujuy: Dpto. Gral. M. Belgrano, Gruta de La Difunta Correa, 24²'55'S, 65²5'27’'W, $07-$ XII-2007, Cabral 829. Prov. Salta: Dpto. Rivadavia, Ruta 81, Km 1692, 23ํ', 62²4’W, 15-I-2002, Solís Neffa 596. 